\title{
Ricci iterations on Kähler classes
}

\author{
Julien Keller
}

October 29, 2018

\begin{abstract}
In this paper we consider the dynamical system involved by the Ricci operator on the space of Kähler metrics. A. Nadel has defined an iteration scheme given by the Ricci operator for Fano manifold and asked whether it has some nontrivial periodic points. First, we prove that no such periodic points can exist. We define the inverse of the Ricci operator and consider the dynamical behaviour of its iterates for a Fano KählerEinstein manifold. In particular we show that the iterates do converge to the Kähler-Ricci soliton for toric manifolds. Finally, we define a finite dimensional procedure to give an approximation of Kähler-Einstein metrics using this iterative procedure and apply it for $\mathbb{P}^{2}$ blown up in 3 points.
\end{abstract}

\section{Introduction}

Let $M$ be a compact Kähler manifold of complex dimension $n$. For any Kähler metric $g$ we let $\omega:=\omega_{g}=\sqrt{-1} / 2 \pi \cdot g_{i \bar{j}}(z) d z^{i} \wedge d \bar{z}^{j}$ denote its corresponding Kähler form, a closed positive $(1,1)$-form on $M$. For a Kähler form $\omega$ such that $[\omega]=c_{1}(M)$ consider the space of strictly $\omega$-plurisubharmonic potentials

$$
K a_{[\omega]}=\left\{\varphi \in \mathrm{C}^{\infty}(M): \omega+\sqrt{-1} \partial \bar{\partial} \varphi>0\right\},
$$

and the space $K a_{c_{1}}$ of Kähler forms cohomologous to $c_{1}(M)$.

In [Na], Nadel considers for a Fano manifold $M$, iterations on $K a_{c_{1}}$ defined inductively using the Ricci operator as follows. Let $K a_{c_{1}}^{(0)}=K a_{c_{1}}$ denote the set of all smooth forms in $c_{1}(M)>0$ and let $\operatorname{Ric}^{(0)}$ be the identity operator. For any Kähler metric we let

$$
\operatorname{Ric}(\omega)=-\partial \bar{\partial} \log \operatorname{det}\left(g_{i \bar{j}}\right)
$$

denote the Ricci form of $\omega$. It is well-defined globally and lies again in the $c_{1}(M)$ class. If it is positive we let $\operatorname{Ric}^{(2)}(\omega)$ denote its Ricci form, and in a similar fashion we define higher powers of the operator as long as the positivity is preserved. The motivation for this construction comes from the simple fact that, when they exist, Kähler-Einstein metrics are by definition fixed points for this iteration process. Nadel asked whether these are all periodic points and proved the absence of periodic points of order 2 and 3. Furthermore he raised the question whether the existence of Kähler-Einstein metrics could be related to this iteration procedure. This question is also very natural. Actually, as we will explain later, one can define the operator $\operatorname{Ric}^{(-1)}$ using Calabi-Yau theorem 
and see its iterations as a kind of naive discretization of the (normalised) KählerRicci flow

$$
\frac{\partial w_{t}}{\partial t}=-\operatorname{Ric}\left(\omega_{t}\right)+\omega_{t}
$$

Thus, we expect some similar properties for the Kähler-Ricci flow and the iterations of the operator $\operatorname{Ric}^{(-1)}$.

We explain now the organization of this paper. We will show that some natural energy functionals are decreasing along these iterations. This will give us a simple proof of the non existence of (non trivial) periodic points and thus answer Nadel's first question. Then we study the question of the existence of periodic points of infinite order. The behaviour of our dynamical system is closely tied with the existence of Kähler-Einstein metrics on the Fano manifold. We generalize Nadel's iteration scheme and define a family of natural operators $\operatorname{Ric}_{\epsilon}, \epsilon \geq 0$ and see that their behaviour is particularly simple when the manifold is Einstein and $\epsilon<1$. In Section 4, we investigate the case of Fano toric manifolds for which we can prove that the iterates of $\operatorname{Ric}^{(-1)}$ do always converge towards a Kähler-Ricci soliton. This also gives a new proof of the existence of Kähler-Ricci solitons on Fano toric manifolds. We shall emphasise the fact that we don't use any flow or continuity method, contrarily to [Zhu2, WZ]. Then we relate the iteration procedure to the notion of canonically balanced metric studied by Donaldson in [Do3]. This gives us an approximation procedure in a finite dimensional setup of the Kähler-Einstein metric when it does exist a priori on a Fano manifold. We apply our techniques to the case of $\mathbb{P}^{2}$ blown up in 3 points and give a numerical approximation of the Kähler-Einstein metric living on it. Finally we discuss the case of the manifolds with negative first Chern class and also non canonical classes.

\section{Positive and negative Ricci iterations}

Our first observation lies in the fact that Nadel's construction can actually be reversed. Let $\alpha$ be any form representing the $c_{1}(M)$ class. By the Calabi-Yau theorem [Ya] there exists a unique Kähler form in $K a_{c_{1}}$, which we denote by $\omega_{1}$, whose Ricci form equals $\alpha$. We define the inverse Ricci operator by

$$
\operatorname{Ric}^{(-1)}(\alpha):=\omega_{1}
$$

Similarly we define higher powers $\operatorname{Ric}^{(-k)}:=\operatorname{Ric}^{(-1)} \circ \cdots \circ \operatorname{Ric}^{(-1)}$, making repeated use of the Calabi-Yau theorem. This leads us to consider for a given Kähler form $\omega_{0}$ the sequence

$$
\operatorname{Ric}\left(\omega_{j+1}\right)=\omega_{j}
$$

for an integer $j \geq 1$. This can be also written

$$
\frac{\omega_{j+1}-\omega_{j}}{(j+1)-j}=-\operatorname{Ric}\left(\omega_{j+1}\right)+\omega_{j+1}
$$

which justifies naively why our iterative procedure (2) should have some similarities with the Kähler-Ricci flow (1). 
For a positive $k$ we will denote $K a_{c_{1}}^{(k)}$ the maximal domain of definition of $\operatorname{Ric}^{(k)}$. We obtain naturally a filtration of $K a_{c_{1}}^{(0)}$. We also let $\overline{K a_{c_{1}}^{(k)}}$ denote the set of all metrics in $K a_{c_{1}}^{(k-1)}$ whose image under $\operatorname{Ric}^{(k-1)}$ is nonnegative.

The advantage of this construction lies in the fact that iterations improve the positivity of $\alpha$. Indeed, if we consider the filtration $\left\{K a_{c_{1}}^{(j)}\right\}_{j>0}$ to measure positivity in some sense, then the image of $\operatorname{Ric}^{(-k)}$ lies in $K a_{c_{1}}^{(k)}$ and we may iterate the inverse Ricci operator to any desired power. Note that this yields homeomorphisms

$$
\operatorname{Ric}^{(k)}:\left(K a_{c_{1}}^{(l)},\|\cdot\|_{\mathrm{C}^{m, \beta}}\right) \rightarrow\left(K a_{c_{1}}^{(l+k)},\|\cdot\|_{\mathrm{C}^{m-2 k, \beta}}\right)
$$

for all $\beta \in(0,1), m, l \in \mathbb{N} \cup\{0\}, k \in \mathbb{Z}$ such that $l+k \geq 0$ and $m \geq 2 k$. We let $K a_{c_{1}}^{(\infty)}$ denote the set of all $L^{\infty}(M)$ limits $\lim _{k \rightarrow \infty} \operatorname{Ric}^{(-k)} \alpha$, for $\alpha \in K a_{c_{1}}^{(0)}$ (when they exist).

Finally, let $G$ be any connected compact subgroup of the $\operatorname{group} \operatorname{Aut}(M, J)$ of holomorphic diffeomorphisms of $(M, J)$. Denote by $K a_{\Omega}(G)$ the space of $G$ invariant Kähler forms in $K a_{\Omega}$. Such forms exists as can be seen by averaging over orbits of $G$ with respect to the Haar measure of $G$. We remark that $\operatorname{Ric}^{(-1)}$ maps $K a_{c_{1}}(G)$ into itself.

\section{Energy functionals on the space of Kähler po- tentials}

In the Sections 2.1 and 2.2, we present some properties of some well-known energy functionals. Let $\Omega \in H^{2}(M, \mathbb{R})$ denote a Kähler class. We call a function $A: K a_{\Omega} \times K a_{\Omega} \rightarrow \mathbb{R}$ an energy functional if it is zero on the diagonal. By an exact energy functional we will mean one which satisfies in addition the cocycle condition (see $[\mathrm{Ma}]$ )

$$
A\left(\omega_{1}, \omega_{2}\right)+A\left(\omega_{2}, \omega_{3}\right)=A\left(\omega_{1}, \omega_{3}\right)
$$

In all the paper, $V$ will denote the volume of the manifold with respect to $[\omega]$, i.e $V=\int_{M} \frac{\omega^{n}}{n !}$. The energy functionals $I, J$, introduced by Aubin in [Au1], are defined for each pair $\left(\omega, \omega_{\varphi}:=\omega+\sqrt{-1} \partial \bar{\partial} \varphi\right)$ by

$$
\begin{gathered}
I\left(\omega, \omega_{\varphi}\right)=\frac{1}{V} \int_{M} \sqrt{-1} \partial \varphi \wedge \bar{\partial} \varphi \wedge \sum_{i=0}^{n-1} \omega^{i} \wedge \omega_{\varphi}^{n-1-i}=\frac{1}{V} \int_{M} \varphi\left(\omega^{n}-\omega_{\varphi}^{n}\right), \\
J\left(\omega, \omega_{\varphi}\right)=\frac{1}{V(n+1)} \int_{M} \sqrt{-1} \partial \varphi \wedge \bar{\partial} \varphi \wedge \sum_{i=0}^{n-1}(n-i) \omega^{i} \wedge \omega_{\varphi}^{n-1-i}
\end{gathered}
$$

We note some of their basic properties for which we refer the reader to [Au1, Si, Ti1]. Note that $I, J$ and $I-J$ are all nonnegative and equivalent. One may also define them via a variational formula. Connect each pair $\left(\omega, \omega_{\varphi_{1}}:=\right.$ $\left.\omega+\sqrt{-1} \partial \bar{\partial} \varphi_{1}\right)$ with a piecewise smooth path $\left\{\omega_{\varphi_{t}}\right\}$. Then, e.g. for $I-J$, we have for any such path

$$
(I-J)\left(\omega, \omega_{\varphi_{1}}\right)=-\frac{1}{V} \int_{[0,1] \times M} \varphi_{t} \Delta_{t} \dot{\varphi}_{t} \omega_{\varphi_{t}}^{n} \wedge d t .
$$




\subsection{The $F_{\mu}$ functional}

Let

$$
F^{0}\left(\omega_{0}, \varphi\right)=-(I-J)\left(\omega_{0}, \omega_{\varphi}\right)-\frac{1}{V} \int_{M} \varphi \omega_{\varphi}^{n}
$$

For a Kähler manifold of positive or negative Chern class define the following functional on $K a_{\mu c_{1}} \times K a_{\mu c_{1}}$

$$
F_{\mu}\left(\omega, \omega_{\varphi}\right)=F^{0}(\omega, \varphi)-\mu \log \left(\frac{1}{V} \int_{M} e^{h_{\omega}-\mu \varphi} \omega^{n}\right)
$$

with $\mu= \pm 1$ respectively. For a Kähler manifold of zero Chern class define on $K a_{\Omega} \times K a_{\Omega}$ the functional

$$
F_{0}\left(\omega, \omega_{\varphi}\right)=F^{0}(\omega, \varphi)+\frac{1}{V} \int_{M} \varphi e^{h} \omega^{n}
$$

The critical points of the functionals $F_{\mu}$ are the Kähler-Einstein metrics. They are absolute minima [Di, Ti1]. Indeed the second variation of $F$ at a critical point in the direction of the plane spanned by $\psi_{1}, \psi_{2} \in T_{\varphi_{t}} K a_{[\omega]}$ is given by

$$
\frac{1}{V} \int_{M}\left[\frac{1}{2} g_{\varphi_{t}}\left(\nabla \psi_{1}, \nabla \psi_{2}\right)-\mu \psi_{1} \psi_{2}\right] \omega_{\varphi_{t}}^{n}, \quad \mu= \pm 1,0
$$

This is seen to be a strictly positive $(0,2)$-tensor on $K a_{[\omega]}$ for $\mu \leq-1$. For $\mu=1$ it is nonnegative and vanishes precisely when $\psi_{1}$ and $\psi_{2}$ are proportional and eigenfunctions of eigenvalue -1 of $\Delta_{\bar{\partial}}$ (see [Ti1, p.64]). In that case this infinitesimal variation corresponds to holomorphic automorphisms and to moving within the set of Kähler-Einstein forms.

\section{$2.2 \quad$ K-energy and $E_{k}$ functionals}

The Chen-Tian energy functionals $E_{k}, k=0, \ldots, n$, are defined in a similar manner by

$$
\begin{aligned}
E_{k}\left(\omega, \omega_{\varphi_{1}}\right)= & \frac{(k+1)}{V} \int_{[0,1] \times M} \Delta_{\varphi_{t}} \dot{\varphi}_{t} \operatorname{Ric}\left(\omega_{\varphi_{t}}\right)^{k} \wedge \omega_{\varphi_{t}}^{n-k} \wedge d t \\
& -\frac{(n-k)}{V} \int_{[0,1] \times M} \dot{\varphi}_{t}\left(\operatorname{Ric}\left(\omega_{\varphi_{t}}\right)^{k+1}-\mu_{k} \omega_{\varphi_{t}}^{k+1}\right) \omega_{\varphi_{t}}^{n-1-k} d t,(8) \\
\mu_{k}= & \frac{c_{1}(M)^{k+1} \cup[\omega]^{n-k-1}([M])}{[\omega]^{n}([M])}
\end{aligned}
$$

This gives rise to well-defined exact energy functionals independent of the choice of path [CT]. The K-energy, $E_{0}$, was introduced by Mabuchi [Ma]. The following formula is taken from [Ti0, Section 7.2]. For the derivation (for any Kähler class) we refer to [Ch] where an equivalent expression is given.

Proposition 2.1. Let $h$ be a function satisfying Ric $\omega-\mu \omega=\sqrt{-1} \partial \bar{\partial} h$. One has

$$
E_{0}\left(\omega, \omega_{\varphi}\right)=\frac{1}{V} \int_{M} \log \frac{\omega_{\varphi}^{n}}{\omega^{n}} \omega_{\varphi}^{n}-\mu(I-J)\left(\omega, \omega_{\varphi}\right)+\frac{1}{V} \int_{M} h\left(\omega^{n}-\omega_{\varphi}^{n}\right)
$$


Remark that the $E_{k}$ functionals vanish on pairs joined by a one parameter subgroup of automorphisms through the identity [CT, Corollary 5.5].

Definition 2.1. We say that an exact functional $T$ is bounded from below if $T\left(\omega, \omega_{\varphi}\right) \geq C$ for every $\omega_{\varphi} \in K a_{\Omega}$. We say it is proper on $K a_{\Omega}(G)$ (in the sense of Tian) if there exists a function $\rho: \mathbb{R} \rightarrow \mathbb{R}$ satisfying $\lim _{s \rightarrow \infty} \rho(s)=\infty$ such that $T\left(\omega, \omega_{\varphi}\right) \geq \rho\left((I-J)\left(\omega, \omega_{\varphi}\right)\right)$ for every $\omega_{\varphi} \in K a_{\Omega}(G)$.

This is well-defined, in other words depends only on $[\omega]$ since the failure of $I-J$ to satisfy the cocycle condition is under control with respect to the two base metrics,

$$
(I-J)\left(\omega, \omega_{\varphi_{2}}\right)-(I-J)\left(\omega_{\varphi_{1}}, \omega_{\varphi_{2}}\right)=(I-J)\left(\omega, \omega_{\varphi_{1}}\right)-\frac{1}{V} \int_{M} \varphi_{1}\left(\omega_{\varphi_{2}}^{n}-\omega_{\varphi_{1}}^{n}\right) .
$$

\subsection{A lower bound for the energy functionals}

We now study the iterations of the $\mathrm{Ric}^{-1}$ operator for Fano manifolds by analysing the behaviour of the functionals that we have introduced. Firstly, we recall a result of non-negativity of Bando and Mabuchi,

Theorem 2.1. [BM, Theorem A], [Ba, Theorem 1], [SW1, Theorem 1.2]. Let $\left(M, \omega_{K E}\right)$ be a Fano Kähler-Einstein manifold. Then for $i=0,1$,

$$
E_{i}\left(\omega_{K E}, \omega\right) \geq 0
$$

for all $\omega \in K a_{c_{1}}$ with equality if and only if $\omega$ is Kähler-Einstein. In that case there exists a holomorphic automorphism homotopic to the identity $h$ such that $h^{\star} \omega_{K E}=\omega$.

We give a sketch of the proof for $i=0$ with an emphasis on the features that will be useful in later sections. Consider the deformation $\left\{\omega_{\varphi_{t}}\right\} \subseteq K a_{c_{1}}$ constructed from two paths, solutions of the following Monge-Ampère equations

$$
\begin{aligned}
\omega_{\varphi_{t}}^{n} & =e^{t f+c_{t}} \omega^{n}, t \in[0,1] \\
& =e^{f-(t-1) \varphi_{t}} \omega^{n}, t \in[1,2]
\end{aligned}
$$

where Ric $\omega-\omega=\sqrt{-1} \partial \bar{\partial} f$ with the normalisations

$$
\int_{M} e^{t f+c_{t}} \omega^{n}=\int_{M} e^{f-(t-1) \varphi_{t}} \omega^{n}=V .
$$

Note that the first path is the one used in Yau's continuity method proof [Ya]. It connects any point $\omega$ in $K a_{c_{1}}$ to $\operatorname{Ric}^{(-1)} \omega$ in $K a_{c_{1}}^{(2)}$. The second path, introduced by Aubin in [Au1], is used to connect any point in $K a_{c_{1}}^{(2)}$ to a Kähler-Einstein metric.

The existence of the first path is equivalent to the Calabi-Yau theorem. The second path may not exist in the presence of nontrivial holomorphic vector fields but Bando and Mabuchi show that arbitrarily close to $\omega$ in the $\mathrm{C}^{\infty}(M)$ topology there exist metrics for which such a path exists. Since the K-energy is continuous this will be sufficient for the argument (Cf. [BM], [SW1, Section 3]).

Now, for $t \in[0,1]$ one has

$$
\operatorname{Ric} \omega_{\varphi_{t}}=(1-t) \operatorname{Ric} \omega+t \omega
$$


and

$$
\Delta_{t} \dot{\varphi}_{t}=f+\dot{c}_{t}
$$

hence

$$
\begin{aligned}
\frac{d}{d t} E_{0}\left(\omega_{K E}, \omega_{\varphi_{t}}\right) & =-\frac{1}{V} \int_{M} \dot{\varphi}_{t}\left(\operatorname{Ric} \omega_{\varphi_{t}}-\omega_{\varphi_{t}}\right) \wedge n \omega_{\varphi_{t}}^{n-1} \\
& =-\frac{1}{V} \int_{M} \dot{\varphi_{t}}\left((1-t) \sqrt{-1} \partial \bar{\partial} f-\sqrt{-1} \partial \bar{\partial} \omega_{\varphi_{t}}\right) \wedge n \omega_{\varphi_{t}}^{n-1} \\
& =-(1-t) \frac{1}{V} \int_{M} \dot{\varphi}_{t}\left(\Delta_{\omega_{\varphi_{t}}} \dot{\varphi}_{t}\right)^{2} \omega_{\varphi_{t}}^{n}-\frac{d}{d t}(I-J)\left(\omega, \omega_{\varphi_{t}}\right)
\end{aligned}
$$

with $t \in[0,1]$, from which we conclude

$$
E_{0}\left(\omega_{K E}, \omega_{\varphi_{1}}\right) \leq E_{0}\left(\omega_{K E}, \omega\right) .
$$

Next, for $t \in[1,2]$

$$
\operatorname{Ric} \omega_{\varphi_{t}}=(2-t) \omega+(t-1) \omega_{\varphi_{t}}
$$

and

$$
\Delta_{t} \dot{\varphi}_{t}=-\varphi_{t}+t \dot{\varphi}_{t},
$$

hence

$$
\begin{aligned}
\frac{d}{d t} E_{0}\left(\omega_{K E}, \omega_{\varphi_{t}}\right) & =-\frac{1}{V} \int_{M} \dot{\varphi}_{t}\left(-(2-t) \sqrt{-1} \partial \bar{\partial} \omega_{\varphi_{t}}\right) \wedge n \omega_{\varphi_{t}}^{n-1} \\
& =-(2-t) \frac{d}{d t}(I-J)\left(\omega_{K E}, \omega_{\varphi_{t}}\right) \leq 0 \\
& =-(2-t) \frac{1}{V} \int_{M}\left(\left(\Delta_{t} \dot{\varphi}_{t}\right)^{2}+t\left|\partial \dot{\varphi}_{t}\right|_{t}^{2}\right) \omega_{\varphi_{t}}^{n} \leq 0,
\end{aligned}
$$

where $t \in[1,2]$. The theorem now follows in this case. Song and Weinkove extended this argument to $E_{1}$ using two detailed computations. The first shows that while $E_{k}$ may not necessarily be monotone (when the path exists), one still has $E_{k}\left(\omega_{K E}, \omega_{\varphi_{1}}\right) \geq E_{k}\left(\omega_{K E}, \omega_{\varphi_{2}}\right)=0$. In other words,

Theorem 2.2. [SW1, Theorem 1.1] Let $\left(M, \omega_{K E}\right)$ be a Fano Kähler-Einstein manifold. Then for any $\omega \in \overline{K a_{c_{1}}^{(2)}}$ and for each $k=0, \ldots, n$ one has

$$
E_{k}\left(\omega_{K E}, \omega\right) \geq 0
$$

with equality if and only if $\omega$ is Kähler-Einstein and $h^{\star} \omega_{K E}=\omega$ with $h$ a biholomorphism homotopic to the identity.

The second calculation shows that when $k=1$, one has

$$
E_{1}\left(\omega_{\varphi_{1}}, \omega\right) \geq E_{1}\left(\omega_{0}, \omega\right)=0
$$

Explicitly, their computation shows that

$$
\begin{aligned}
E_{k}\left(\omega_{\varphi_{1}}, \omega\right)= & \frac{1}{V} \int_{M} \sqrt{-1} \partial \varphi_{1} \wedge \bar{\partial} \varphi_{1} \wedge \sum_{i=0}^{n-1} a_{i} \omega^{i} \wedge \omega_{\varphi_{1}}^{n-1-i} \\
& +(k+1) \frac{1}{V} \int_{M \times[0,1]}(1-t)\left(\Delta_{\omega_{\varphi_{t}}} \dot{\varphi}_{t}\right)^{2} \omega_{\varphi_{t}}^{n} \wedge d t \\
& -\frac{1}{V} \int_{M} \sum_{i=1}^{k}\left(\begin{array}{c}
i+1 \\
k+1
\end{array}\right) f(\sqrt{-1} \partial \bar{\partial} f)^{i} \wedge \omega^{n-i},
\end{aligned}
$$

with $a_{i}=\frac{(n-k)(i+1)}{n+1}$ if $0 \leq i \leq k-1$ and $a_{i}=\frac{(k+1)(n-i)}{n+1}$ if $k \leq i \leq n$. Since the last term is positive on $K a_{c_{1}}$ for $k=1$ they conclude their proof. 


\subsection{A system of Monge-Ampère equations}

Now, let $\omega=\omega_{0}$ denote an initial Kähler metric for our iterations. We present the iterative procedure defined by (2) in terms of Monge-Ampère equations. Let $\varphi_{1}$ be a Kähler potential with

$$
\operatorname{Ric}\left(\omega_{0}+\sqrt{-1} \partial \bar{\partial} \varphi_{1}\right)=\omega_{0}
$$

Set the Ricci deviation $h:=h_{\omega_{0}}$ of $\omega_{0}$ as $\sqrt{-1} \partial \bar{\partial} h=\operatorname{Ric} \omega_{0}-\omega_{0}$. The function $h$ thus given is for the moment determined only up to a an additive constant. The equation then becomes

$$
-\sqrt{-1} \partial \bar{\partial} \log \operatorname{det} g_{\varphi_{1}}=\omega_{0}=-\sqrt{-1} \partial \bar{\partial} \log \operatorname{det} g-\sqrt{-1} \partial \bar{\partial} h
$$

or

$$
\sqrt{-1} \partial \bar{\partial} \log \frac{\omega_{\varphi_{1}}^{n}}{\omega^{n}}=\sqrt{-1} \partial \bar{\partial} h
$$

that is

$$
\omega_{\varphi_{1}}^{n}=e^{h} \omega^{n}
$$

together with the volume normalisation

$$
\frac{1}{V} \int_{M} e^{h} \omega^{n}=1
$$

This determines $\varphi_{1}$ only up to a constant, which will be fixed in the the next step. Put $\omega_{1}=\omega_{\varphi_{1}}$. In the second step we solve

$$
\operatorname{Ric}\left(\omega_{1}+\sqrt{-1} \partial \bar{\partial} \varphi_{2}\right)=\omega_{1}
$$

and $\omega_{1}-\operatorname{Ric} \omega_{1}=\omega_{1}-\omega_{0}=\sqrt{-1} \partial \bar{\partial} \varphi_{1}$. The Monge-Ampère equation is now

$$
\omega_{\varphi_{1}+\varphi_{2}}^{n}=e^{-\varphi_{1}} \omega_{\varphi_{1}}^{n}=e^{h-\varphi_{1}} \omega^{n},
$$

with $\varphi_{1}$ determined uniquely by

$$
\frac{1}{V} \int_{M} e^{h-\varphi_{1}} \omega^{n}=1
$$

Iterating this procedure we have $\operatorname{Ric}^{(-l)} \omega=\omega_{\Sigma_{j=1}^{l} \varphi_{j}}$ for each $l \in \mathbb{N}$ where

$$
\left(\omega+\sqrt{-1} \partial \bar{\partial} \Sigma_{j=1}^{l} \varphi_{j}\right)^{n}=e^{h-\Sigma_{j=1}^{l-1} \varphi_{j}} \omega^{n},
$$

and each of the $\varphi_{j}$ is uniquely determined by

$$
\frac{1}{V} \int_{M} e^{h-\Sigma_{j=1}^{l-1} \varphi_{j}} \omega^{n}=1 .
$$

From now on we set

$$
\Phi_{l}=\Sigma_{j=1}^{l} \varphi_{j}
$$

and

$$
\omega_{l}=\omega_{\Phi_{l}}=\omega_{0}+\sqrt{-1} \partial \bar{\partial} \Phi_{l} .
$$




\subsection{Monotonicity of the energy functionals}

The following proposition describes the monotonicity of the K-energy and $F_{\mu}$ along the iteration. Note that the equivalent for the Kähler-Ricci flow is wellknown.

Proposition 2.2. Let $\mu=1$. Then

$$
\begin{aligned}
& E_{0}\left(\omega, \omega_{l}\right)=-(I-J)\left(\omega, \omega_{l}\right)-\frac{1}{V} \int_{M} \Phi_{l-1} \omega_{l}^{n}+\frac{1}{V} \int_{M} h \omega^{n} \leq 0, \\
& F_{\mu}\left(\omega, \omega_{l}\right)=F^{0}\left(\omega, \Phi_{l}\right) \leq 0 \\
& E_{1}\left(\omega, \omega_{l}\right) \leq 0
\end{aligned}
$$

with equality if and only if $\omega$ is Kähler-Einstein. Furthermore all the $E_{k}$ with $k=2, \ldots, n$ decrease along the iteration starting from the second iteration.

Proof. To show the first inequality we note that

$$
\begin{aligned}
E_{0}\left(\omega_{k-1}, \omega_{k}\right) & =\frac{1}{V} \int_{M}-\varphi_{k-1} \omega_{k}^{n}-(I-J)\left(\omega_{k-1}, \omega_{k}\right)-\frac{1}{V} \int_{M} \varphi_{k-1}\left(\omega_{k-1}^{n}-\omega_{k}^{n}\right) \\
& =-(I-J)\left(\omega_{k-1}, \omega_{k}\right)-\frac{1}{V} \int_{M} \varphi_{k-1} \omega_{k-1}^{n} .
\end{aligned}
$$

The first term is nonpositive with equality if and only if $\omega_{k}=\omega_{k-1}=\operatorname{Ric} \omega_{k}$, while the second term is nonpositive since

$$
1=\frac{1}{V} \int_{M} \omega_{k}^{n}=\frac{1}{V} \int_{M} e^{-\varphi_{k-1}} \omega_{k-1}^{n} \geq \frac{1}{V} \int_{M}\left(1-\varphi_{k-1}\right) \omega_{k-1}^{n} .
$$

Since

$$
E_{0}\left(\omega, \omega_{l}\right)=\sum_{k=1}^{l} E_{0}\left(\omega_{k-1}, \omega_{k}\right)
$$

the conclusion follows.

The second inequality follows similarly, indeed

$$
F_{1}\left(\omega_{k-1}, \omega_{k}\right)=-(I-J)\left(\omega_{k-1}, \omega_{k}\right)-\frac{1}{V} \int_{M} \varphi_{k} \omega_{k}^{n},
$$

and with (22). The third inequality follows from

$$
E_{1}\left(\omega_{k-1}, \omega_{k}\right)=2 F_{1}\left(\omega_{k-1}, \omega_{k}\right)+\frac{1}{V} \int_{M} \varphi_{k}\left(\omega_{k}^{n}+\omega_{k}^{n-1} \wedge \omega_{k-1}\right)
$$

from the formula relating $F_{1}$ and $E_{k}$. Since both summands are negative, we conclude (note that $\omega_{k-1}-\omega_{k}=-\sqrt{-1} \partial \bar{\partial} \varphi_{k}$ ). Finally, the decrease of the functionals are proved using (15) and (17).

Remark 2.1. For the rest of the energy functionals, we get by direct computations that with respect to a Kähler-Einstein metric $\omega$,

$$
\begin{aligned}
E_{k}\left(\omega, \omega_{\varphi}\right) & =(k+1) F_{1}\left(\omega, \omega_{\varphi}\right)-\frac{1}{V} \int_{M} f_{\omega_{\varphi}}\left(\omega_{\varphi}^{n}+\ldots+\omega_{\varphi}^{n-k} \wedge\left(\operatorname{Ric} \omega_{\varphi}\right)^{k}\right) \\
& =(k+1) F_{1}\left(\omega, \omega_{\varphi}\right)-B_{k}\left(\omega, \omega_{\varphi}\right) .
\end{aligned}
$$


where Ric $\omega_{\varphi}=\omega_{\varphi}+\sqrt{-1} \partial \bar{\partial} f_{\omega_{\varphi}}$. Note that when the Ricci curvature is positive we have

$$
\begin{aligned}
& B_{k}\left(\omega, \omega_{\varphi}\right)=\frac{1}{V} \int_{M} f_{\omega_{\varphi}}\left(\omega_{\varphi}+\sqrt{-1} \partial \bar{\partial} f_{\omega_{\varphi}}\right) \wedge\left(\omega_{\varphi}^{n-1}+\ldots+\omega_{\varphi}^{n-k} \wedge\left(\operatorname{Ric} \omega_{\varphi}\right)^{k-1}\right) \\
& =-\frac{1}{V} \int_{M} \sqrt{-1} \partial f_{\omega_{\varphi}} \wedge \bar{\partial} f_{\omega_{\varphi}} \wedge\left(\omega_{\varphi}^{n-1}+\ldots+\omega_{\varphi}^{n-k} \wedge\left(\operatorname{Ric} \omega_{\varphi}\right)^{k-1}\right) \\
& +\frac{1}{V} \int_{M} f_{\omega_{\varphi}} \omega_{\varphi} \wedge\left(\omega_{\varphi}^{n-1}+\ldots+\omega_{\varphi}^{n-k} \wedge\left(\operatorname{Ric} \omega_{\varphi}\right)^{k-1}\right) \\
& \leq B_{0}\left(\omega, \omega_{\varphi}\right) \leq 0 \text {. }
\end{aligned}
$$

\section{The dynamics of the Ricci operator}

We are now ready to answer the question raised by Nadel [Na]. Note that some of the results presented in that section were discussed in details by the author and $\mathrm{Y}$. Rubinstein $[\mathrm{Ru}]$ during the period ${ }^{1}$ of their collaboration.

Theorem 1. Let $(M, \omega)$ be a Kähler manifold with positive first Chern class and assume that $\operatorname{Ric}^{(k)}(\omega)=\omega$ for some $k \in \mathbb{N}$. Then $\omega$ is Kähler-Einstein.

Proof. Note that the nonexistence of fixed points of negative order implies that of positive order, and reversely. Therefore assume that for some $\omega \in K a_{c_{1}}$ and some $l \in \mathbb{N}$ one has $\operatorname{Ric}^{(-l)}(\omega)=\omega$. By the cocycle condition we therefore have

$$
0=E_{0}\left(\omega, \operatorname{Ric}^{(-l)} \omega\right)=\sum_{i=0}^{l-1} E_{0}\left(\operatorname{Ric}^{(-i)} \omega, \operatorname{Ric}^{(-i-1)} \omega\right) .
$$

On the other hand, from the first part of (11)

$E_{0}\left(\omega, \operatorname{Ric}^{(-1)} \omega\right)=-\frac{1}{V} \int_{M \times[0,1]}(1-t)\left(\Delta_{\omega_{\varphi_{t}}} \dot{\varphi}_{t}\right)^{2} \omega_{\varphi_{t}}^{n} \wedge d t-(I-J)\left(\omega, \operatorname{Ric}^{(-1)} \omega\right)$

Thus $E_{0}\left(\omega, \operatorname{Ric}^{(-1)} \omega\right) \leq 0$, with equality if and only if $\operatorname{Ric}^{(-1)} \omega=\omega$. Therefore each of the terms in (24) must vanish identically and we conclude that $(M, \omega)$ is Kähler-Einstein.

Proposition 3.1. Let $M$ be as above and assume that $\omega \in K a_{c_{1}}^{(k)}$ for all $k \in \mathbb{N}$ and that $\omega$ is not Kähler-Einstein. Then $\lim _{k \rightarrow \infty} \operatorname{Ric}^{(k)} \omega$ does not exist in $K a_{c_{1}}^{(0)}$.

Proof. If $\omega_{\infty}=\lim _{l \rightarrow \infty} \operatorname{Ric}\left({ }^{(l)} \omega\right.$ exists and is smooth it satisfies Ric $\omega_{\infty}=\omega_{\infty}$. But $E_{0}\left(\omega, \omega_{\infty}\right)>0$ contradicting (2.1).

Let $G$ denote the Green function for $\Delta=\Delta_{\bar{\partial}}$ with respect to $(M, \omega)$ with $\int_{M} G(x, y) \omega^{n}(y)=0$ and $A(\omega)=-\inf G$ such that

$$
f(x)-\frac{1}{V} \int_{M} f \omega^{n}=-\frac{1}{V} \int_{M} G(x, y) \Delta f(y) \omega^{n}(y), \quad \forall f \in \mathrm{C}^{\infty}(M) .
$$

Then, one has the following estimate due to Bando and Mabuchi.

\footnotetext{
${ }^{1}$ September 2003 to December 2006.
} 
Theorem 3.1. [BM] One has

$$
A(\omega) \leq \frac{c_{n}}{2} \operatorname{diam}(M, \omega)^{2} .
$$

If $\operatorname{Ric}(\omega) \geq \epsilon \omega$ for some $\epsilon>0$ then $\operatorname{diam}(M, \omega)^{2} \leq \frac{\pi^{2}(2 n-1)}{\epsilon}$ by Myers' theorem.

As an immediate corollary we have

Lemma 3.1. Let $M$ be a Fano manifold. Assume that the K-energy is proper. Let $\left(\omega_{l}\right)_{l \in \mathbb{N}}$ be a sequence of Kähler forms on which the K-energy is bounded from above and such that there exists $l_{0}\left(\omega_{0}\right) \in \mathbb{N}$ and $\epsilon>0$ with Ric $\omega_{l} \geq \epsilon \omega_{l}, \forall l \geq l_{0}$. Then there exists a constant $C_{1}$ depending only on $\left(M, \omega_{0}\right)$ such that

$$
\left\|\Phi_{l}\right\|_{L^{\infty}(M, \omega)} \leq C_{1}, \quad \forall l \in \mathbb{N}
$$

where $\omega_{l}=\omega_{0}+\sqrt{-1} \partial \bar{\partial} \Phi_{l}$ and $\frac{1}{V} \int_{M} e^{h-\Phi_{l}} \frac{\omega_{0}^{n}}{n !}=1$.

Proof. Let $G_{l}$ be the Green function for $\Delta_{l}=\Delta_{\bar{\partial}, \omega_{l}}$ (i.e., the Laplacian with respect to $\left.\left(M, \omega_{l}\right)\right)$ satisfying $\int_{M} G_{l}(x, y) \omega_{l}^{n}(y)=0$. Set $A_{l}=-\inf _{M \times M} G_{l}$.

Since $-n<\Delta_{1} \Phi_{l}$ and $n>\Delta_{l} \Phi_{l}$ the Green formula gives

$$
\begin{aligned}
& \Phi_{l}(x)-\frac{1}{V} \int_{M} \Phi_{l} \omega_{0}^{n}=-\frac{1}{V} \int_{M} G_{1}(x, y) \Delta \Phi_{l}(y) \omega_{0}^{n}(y) \leq n A_{1}, \\
& \Phi_{l}(x)-\frac{1}{V} \int_{M} \Phi_{l} \omega_{l}^{n}=-\frac{1}{V} \int_{M} G_{l}(x, y) \Delta \Phi_{l}(y) \omega_{l}^{n}(y) \geq-n A_{l} .
\end{aligned}
$$

Hence

$$
\operatorname{osc}_{M} \Phi_{l} \leq n\left(A_{1}+A_{l}\right)+I\left(\omega, \omega_{l}\right) .
$$

Since $E_{0}$ is proper on $K a_{c_{1}}(G)$ in the sense of Tian, if $E_{0}\left(\omega_{0}, \cdot\right)$ is uniformly bounded from above on a subset of $K a_{c_{1}}(G)$ so is $I(\omega, \cdot)$. We conclude that $I\left(\omega_{0}, \omega_{l}\right)$ is uniformly bounded independently of $l$. Finally, the Proposition follows from Theorem 3.1 which provides a uniform bound for $A_{l}$.

As a consequence of the properness of the K-energy for Fano Einstein manifolds [Ti1, PSSW] we obtain the following,

Corollary 3.1. Let $M$ be a Fano Einstein manifold with no nontrivial holomorphic vector field. Consider the sequence of Kähler metrics $\left(\omega_{l}\right)_{l \in \mathbb{N}}$ defined by the system of Monge-Ampère equations (19) and assume that for $l$ sufficiently large there exists a constant $\epsilon>0$ with

$$
\operatorname{Ric}\left(\omega_{l}\right) \geq \epsilon \omega_{0} .
$$

Then $\omega_{l}$ converges to the Kähler-Einstein metric when l tends to infinity.

Finally, inspired by $[\mathrm{Pa}]$, we derive the following,

Proposition 3.2. Let $M$ be a Fano manifold with $G$ a maximal compact subgroup of $\operatorname{Aut}(M, J)$. Consider the sequence of $G$-invariant Kähler metrics $\omega_{l}$ defined by the system of Monge-Ampère equations (19). Assume that there exists a constant $1>\kappa>0$ such that for $l$ sufficiently large,

$$
(2-\kappa) \omega_{0}^{n} \geq \omega_{l}^{n} \geq \kappa \omega_{0}^{n}
$$

where $\omega_{0}$ is $G$-invariant Kähler metric. Then $M$ is Kähler-Einstein and $\omega_{l}$ converges to a $G$-invariant Kähler-Einstein metric when l tends to infinity. 
Proof. Thanks to the proof of Lemma 3.1 and Theorem 3.1, we are reduced to prove an upper bound for $I\left(\omega, \omega_{l}\right)$. But if we denote $\Phi_{l}^{+}(x)=\sup \left\{0, \Phi_{l}(x)\right\}$ and $\Phi_{l}^{-}(x)=\inf \left\{0, \Phi_{l}(x)\right\}$, we obtain

$$
\begin{aligned}
I\left(\omega, \omega_{l}\right) & \leq \frac{1}{V} \int_{\left\{\omega_{l}^{n} \geq \omega_{0}^{n}\right\}}\left(-\Phi_{l}^{-}\right)\left(\omega_{l}^{n}-\omega_{0}^{n}\right)+\frac{1}{V} \int_{\left\{\omega_{0}^{n} \geq \omega_{l}^{n}\right\}}\left(\Phi_{l}^{+}\right)\left(\omega_{0}^{n}-\omega_{l}^{n}\right) \\
& \leq(1-\kappa) \frac{1}{V} \int_{M}\left(\Phi_{l}^{+}-\Phi_{l}^{-}\right) \omega_{0}^{n} \\
& \leq(1-\kappa) \operatorname{osc}_{M} \Phi_{l}
\end{aligned}
$$

Together with

$$
\operatorname{Osc}_{M} \Phi_{l} \leq n\left(A_{1}+A_{l}\right)+I\left(\omega, \omega_{l}\right) .
$$

this gives us to the $\mathrm{C}^{0}$ bound for $\Phi_{l}$. Now it is a standard argument of MongeAmpère equations to get the convergence in the $\mathrm{C}^{\infty}$ topology.

For a Kähler manifold $M$, we consider the family of Monge-Ampère equations(11). We introduce the Aubin operators Ric $_{\epsilon}$ by setting

$$
\operatorname{Ric}_{\epsilon}(\omega)=\omega_{\varphi_{1+\epsilon}}
$$

for each $\epsilon \in[0,1]$ such that $\varphi_{1+\epsilon}$, solution of (11), exists. Note that $\operatorname{Ric}_{0}(\omega)=$ $\operatorname{Ric}^{(-1)} \omega$ and $\operatorname{Ric}_{1}(\omega)=\omega_{K E}$. Formally, one can think

$$
\operatorname{Ric}_{\epsilon}=\left(\frac{1}{1-\epsilon}(\operatorname{Ric}-\epsilon I d)\right)^{-1}
$$

and that we have defined the following sequence of Monge-Ampère equations

$$
\left(\omega_{0}+\sqrt{-1} \partial \bar{\partial} \Phi_{j}\right)^{n}=e^{(\epsilon-1) \Phi_{j-1}-\epsilon \Phi_{j}}\left(\omega_{0}+\sqrt{-1} \partial \bar{\partial} \Phi_{j-1}\right)^{n}
$$

Let $G \subseteq \operatorname{Aut}(M, J)$ be as before. Then from uniqueness of solutions of the family of the Monge-Ampère equations (11) we conclude that $\operatorname{Ric}_{\epsilon}$ maps $K a_{c_{1}}(G)$ into itself.

We recall the definition of $\alpha$-invariant introduced by Tian.

$$
\alpha_{M}=\sup \left\{\alpha \geq 0: \sup _{\varphi \in K a_{c_{1}}} \int_{M} e^{-\alpha \varphi} \omega^{n}<\infty\right\} .
$$

In [Ti0] it is proved that $\alpha_{M}$ is a positive holomorphic invariant of Fano manifolds. Regarding the existence of the Aubin operators we state the following

Proposition 3.3.

(i) [Ti0] Assume that $M$ is Fano. Then the operators Ric $_{\epsilon}$ exist for all $\epsilon \in$ $\left[0, \min \left\{1, \frac{n+1}{n} \alpha_{M}\right\}\right)$.

(ii) [BM, Theorem 5.7] Assume in addition that the K-energy is bounded from below. Then the operators Ric $_{\epsilon}$ exist for any $\epsilon \in[0,1)$.

We now recover by a conceptually simpler method a theorem of Tian [Ti1].

Corollary 3.2. Let $M$ be a Fano manifold. Let $G$ be a maximal compact subgroup of $\operatorname{Aut}(M, J)$ and assume that the $K$-energy is proper on $K a_{c_{1}}(G)$ and let $\epsilon \in(0,1)$. Then there exist $G$-invariant Kähler-Einstein metrics. All such metrics are the limit points of the iterates of $\operatorname{Ric}_{\epsilon}$ on $K a_{c_{1}}(G)$ in the $C^{\infty}(M)$ topology. 
Proof. Since the K-energy is proper on $K a_{c_{1}}(G)$ and in particular bounded from below, $\operatorname{Ric}_{\epsilon}$ are defined for each $\epsilon \in(0,1)$. As before we obtain (27) with $\omega_{l}=$ $\operatorname{Ric}_{\epsilon}^{(l)}(\omega)$ with each $\omega_{l}$ in $K a_{c_{1}}(G)$. Since by (15) and (16) the K-energy decreases along iterates we still have a uniform bound on $I$ along the orbits. By Theorem 3.1 we also have a uniform bound (depending on $\epsilon$ ) on $A\left(\operatorname{Ric}_{\epsilon}^{(l)}(\omega)\right.$ ). For each $\beta \in(0,1)$ the $\mathrm{C}^{2, \beta}(M)$-estimates now follow from [Au3, Chapter 7] and the higher order derivatives follow from these, by bootstrapping. We may therefore extract a converging subsequence $\operatorname{Ric}_{\epsilon}^{\left(l_{j}\right)}(\omega)$ in the $\mathrm{C}^{2, \beta}(M, \omega)$-topology whose limit $\omega_{\infty}$ lies in $\mathrm{C}^{2}(M)$. Moreover, since the K-energy is bounded from below we have

$$
\lim _{j \rightarrow \infty} E_{0}\left(\operatorname{Ric}_{\epsilon}^{\left(l_{j}\right)}(\omega), \operatorname{Ric}_{\epsilon}^{\left(l_{j+1}\right)}(\omega)\right)=\lim _{j \rightarrow \infty} \sum_{k=l_{j}}^{l_{j+1}-1} E_{0}\left(\operatorname{Ric}_{\epsilon}^{(k)}(\omega), \operatorname{Ric}_{\epsilon}^{(k+1)}(\omega)\right)=0
$$

As each of the summands is nonpositive one has $E_{0}\left(\omega_{\infty}, \operatorname{Ric}_{\epsilon}\left(\omega_{\infty}\right)\right)=0$ and it follows that $\omega_{\infty} \in K a_{c_{1}}(G)$ is smooth and Kähler-Einstein by (15) and (16). Since this is true for each converging subsequence we conclude that, in fact, the sequence of iterates itself converges.

In fact we also get,

Theorem 2. Let $M$ be a Kähler-Einstein manifold with positive first Chern class. Let $G$ be a maximal compact subgroup of $\operatorname{Aut}(M, J)$.

Then for any $\epsilon \in(0,1), \omega \in K a_{c_{1}}(G)$ there exists a biholomorphism $h$ of $(M, J)$ such that one has $\lim _{l \rightarrow \infty} \operatorname{Ric}_{\epsilon}^{(l)} \omega=h^{\star} \omega_{K E}$ in the $C^{\infty}(M)$-topology for some $G$-invariant Kähler-Einstein form $\omega_{K E}$.

\section{The case of toric Fano manifolds}

On a toric Kähler manifold $M$ with positive first Chern class, a result of X-J. Wang and X. Zhu [WZ] asserts that there does always exist a Kähler-Ricci soliton, which is unique up to holomorphic automorphims. A Kähler-Ricci soliton is a pair $\left(X, \omega_{g}\right)$ where $X$ is a holomorphic vector field on $M$ and $\omega=\omega_{g}$ a Kähler form that satisfies

$$
\operatorname{Ric}(\omega)=\omega+L_{X}(\omega)
$$

where $L_{X}$ is the Lie derivative along $X$. In fact, the Kähler-Ricci soliton in the toric case is a genuine Kähler-Einstein metric if and only if the Futaki invariant vanishes which implies that the holomorphic automorphism group of the manifold is reductive.

Given a nontrivial holomorphic vector field $X$ on $M$, it is well known by Hodge theory that there exists a unique smooth complex-valued function $\theta_{X}$ such that

$$
i_{X}(\omega)=\sqrt{-1} \bar{\partial} \theta_{X}
$$

with $\int_{M} e^{\theta_{X}} \omega^{n}=V$. Let $h \in \mathrm{C}^{\infty}(M)$ be the Ricci deviation of $\omega$ determined by $\frac{1}{V} \int_{M} e^{h} \omega^{n}=1$. From [WZ, Lemma 2.1], there exists a unique holomorphic vector field $X$ such that

$$
F_{X}(v):=\int_{M} v\left(h-\theta_{X}\right) e^{\theta_{X}} \omega^{n}=0
$$


for all holomorphic vector field $v$. Note that $F_{X}$ is actually independent of the choice of $\omega$. For such a vector field $X$, to find a Kähler-Ricci soliton $(X, \omega+$ $\sqrt{-1} \partial \bar{\partial} \varphi$ ) satisfying (30) is equivalent to solve the Monge-Ampère equation

$$
(\omega+\sqrt{-1} \partial \bar{\partial} \varphi)^{n}=e^{h-\theta_{X}-X(\varphi)-\varphi} \omega^{n}
$$

In [Zhu1], an existence result (proved by a continuity method argument) is given for the Monge-Ampère equations of the form

$$
\operatorname{det}\left(g_{i \bar{j}}+\varphi_{i \bar{j}}\right)=e^{\left.f-t \theta_{X}-t X(\varphi)\right)} .
$$

where $f$ is smooth and $t$ varies from 0 to 1 (note that $t=0$ corresponds exactly to Calabi-Yau theorem). This can be seen as consequence of the work of S. Kolodziej [Ko] for solving Monge-Ampère equations, and the following result [Zhu1, Corollary 5.3].

Lemma 4.1. Let $(M, \omega)$ a Kähler manifold with a non-trivial holomorphic vector field $X$. Suppose that $\varphi$ is smooth, $\omega+\sqrt{-1} \partial \bar{\partial} \varphi>0$ and $X(\varphi)$ is a realvalued function. Then there is a uniform constant $C$ independent of $\varphi$ such that $|X(\varphi)|<C$.

Hence, we can define naturally the sequence of Monge-Ampère equations

$$
\left(\omega_{j-1}+\sqrt{-1} \partial \bar{\partial} \varphi_{j}\right)^{n}=e^{h-\theta_{X}-X\left(\varphi_{j}\right)-\varphi_{j-1}} \omega_{j-1}^{n}
$$

which turns out to be equivalent to solve for all $j \geq 1$

$$
\operatorname{Ric}\left(\omega_{j}\right)=\omega_{j-1}+L_{X}\left(\omega_{j}\right)
$$

for $\omega_{j}=\omega_{j-1}+\sqrt{-1} \partial \bar{\partial} \varphi_{j}=\omega_{0}+\sqrt{-1} \partial \bar{\partial} \Phi_{j}$.

The goal of this section is to prove that under this setting, one can always get a $\mathrm{C}^{0}$ estimate for the potentials $\Phi_{j}$, which means that the iteration procedure defined by (31) converges to the unique Kähler-Ricci soliton on the manifold. We follow the techniques developed in [WZ] using real Monge-Ampère equations to derive the uniform estimate for $\Phi_{j}$.

We shall need the following lemma that we will apply with the convex polyhedron $\Omega^{*}$ associated to our toric manifold.

Lemma 4.2. Let $\Omega$ a bounded convex domain in $\mathbb{R}^{n}$. Then there is a unique ellipsoid $\mathcal{E}$ called the minimum ellipsoid of $\Omega$ which attains minimum volume among all ellipsoids containing $\Omega$, such that

$$
\frac{1}{n} \mathcal{E} \subset \Omega \subset \mathcal{E}
$$

where $r \mathcal{E}$ denotes the dilatation of factor $r$ of $\mathcal{E}$ with concentrated factor.

Note that the vector field $X$ can be expressed in the form

$$
X=\sum_{i} c_{i} X_{i}
$$


with $X_{i}=\frac{\partial}{\partial \gamma_{i}}$ for $\left(\gamma_{i}=x_{i}+\sqrt{-1} \theta_{i}\right)_{i=1, . . n}$ affine logarithm coordinates on the maximal torus $T$ of $\operatorname{Aut}(M, J)$. In [WZ, Section 2] it is shown that these constants $c_{i}$ satisfy the relations

$$
\int_{\Omega^{*}} y_{i} e^{\sum_{l=1}^{n} c_{l} y_{l}} d y=0
$$

for all $i=1, . ., n$.. Furthermore, there exists a Kähler form $\omega_{0}$, invariant under the maximal compact abelian subgroup $K_{0}$ of $T$, and determined by a convex function $w_{0}$ on $\mathbb{R}^{n}$, i.e on $T$

$$
\omega_{0}=\sqrt{-1} \partial \bar{\partial} w_{0} .
$$

In all the following, we shall $\operatorname{denote} \operatorname{det}\left(w_{0}\right):=\operatorname{det}\left(\left(w_{0}\right)_{i j}\right)$. We obtain

$$
\omega_{0}^{n}=\frac{1}{\pi^{n}} \operatorname{det}\left(w_{0}\right) d x_{1} \wedge \ldots \wedge d x_{n} \wedge d \theta_{1} \wedge \ldots \wedge d \theta_{n} .
$$

Note that the function $w_{0}$ is determined uniquely by the convex polyhedron $\Omega^{*}$. Indeed, if $\left\{p^{(i)}\right\}_{i=1, . ., m}$ denote the vertices of $\Omega^{*}, w_{0}$ is the convex function defined by $w_{0}=\log \left(\sum_{i=1}^{m} e^{\left\langle p_{i}, x\right\rangle}\right)$. After normalisation and without loss of generality, we can assume $\operatorname{det}\left(w_{0}\right)=e^{-h-w_{0}}$. We initialize our iteration procedure by chosing this way the Kähler form $\omega_{0}$. Let's call $w_{j}=w_{0}+\Phi_{j}$ with $\Phi_{j}$ a $K_{0}$-invariant potential. Now, there exists a constant $c_{X}$ such that

$$
\theta_{X}+X\left(\Phi_{j}\right)=\sum_{l=1}^{n} c_{l} \frac{\partial w_{j}}{\partial x_{l}}+c_{X}
$$

This means that to solve (31) is equivalent to solve the real Monge-Ampère equation on $\mathbb{R}^{n}$,

$$
\operatorname{det}\left(w_{0}+\Phi_{j}\right)=\operatorname{det}\left(w_{j}\right)=e^{-c_{X}-w_{j-1}-\sum c_{l} \frac{\partial w_{j}}{\partial x_{l}}}
$$

Lemma 4.3. Define

$$
m_{j}=\inf _{\mathbb{R}^{n}} w_{j} .
$$

Then there exists a constant $C$ independent of $j$ such that

$$
m_{j} \leq C .
$$

Proof. For an integer $k$, we choose $x_{j, k}$ a point in $\mathbb{R}^{n}$ such that $w_{j}\left(x_{j, k}\right)=m_{j}+k$. We introduce for $j \geq 0$, the set

$$
\begin{aligned}
A_{k, j}=\left\{x \in \mathbb{R}^{n}::\right. & m_{j}+k \leq w_{j}(x) \leq m_{j}+k+1, \\
& \left.m_{j+1}+r_{j+1, k} \leq w_{j+1}(x) \leq m_{j+1}+r_{j+1, k}+1\right\}
\end{aligned}
$$

where $r_{j+1, k}=\left[w_{j+1}\left(x_{j, k}\right)-m_{j+1}\right]+k$. For any integer $k \geq 0, \cup_{0 \leq i \leq k}^{k} A_{i, j}$ is convex. On the other hand, we know that $D \omega_{j}\left(\mathbb{R}^{n}\right)=\Omega^{*}$ and thus $A_{k, j}$ is bounded. Let's define $d_{\max }=\sup _{y \in \Omega^{*}}\left\{c_{l} y_{l}\right\}$. We get on $A_{0, j}$,

$$
\operatorname{det}\left(w_{j+1}\right) \geq e^{-c_{X}-d_{\max }-1} e^{-m_{j}} .
$$

From Lemma 4.2, there exists a linear transformation $y=\operatorname{Tr}(x)$ which leaves the center of the minimum ellipsoid of $A_{0, j}$ invariant such that $B_{R / n} \subset \operatorname{Tr}\left(A_{0, j}\right) \subset$ 
$B_{R}$ for a certain well-chosen constant $R$. Let's try to obtain some information about this radius. Define

$$
v_{j}=\frac{1}{2}\left(e^{-c_{X}-d_{\max }-1}\right)^{\frac{1}{n}} e^{-\frac{m_{j}}{n}}\left(\left|y-y_{j}\right|^{2}-\left(\frac{R}{n}\right)^{2}\right)+m_{j+1}+r_{j+1,0}+1
$$

where $y_{j}$ the center of the minimum ellipsoid of $A_{0, j}$. Then, in $\operatorname{Tr}\left(A_{0, j}\right)$,

$$
\operatorname{det}\left(v_{j}\right)=e^{-c_{X}-d_{\max }-1} e^{-m_{j}}
$$

and of course, $v_{j} \geq w_{j+1}$ on $\partial \operatorname{Tr}\left(A_{0, j}\right)$. The comparison principle for MongeAmpère operator gives that $v_{j} \geq w_{j+1}$ on $\operatorname{Tr}\left(A_{0, j}\right)$. Hence,

$$
\begin{aligned}
m_{j+1}+r_{j+1,0} & \leq w_{j+1}\left(y_{j}\right) \\
& \leq v_{j}\left(y_{j}\right) \\
& =-\frac{1}{2}\left(e^{-c_{X}-d_{\max }-1}\right)^{\frac{1}{n}} e^{-\frac{m_{j}}{n}}\left(\frac{R}{n}\right)^{2}+m_{j+1}+r_{j+1,0}+1
\end{aligned}
$$

This gives us the following inequality with $C_{0}=\sqrt{2} n\left(e^{-c_{X}-d_{\max }-1}\right)^{-\frac{1}{2 n}}$,

$$
R \leq C_{0} e^{\frac{m_{j}}{2 n}}
$$

On the other hand, since $\operatorname{Tr}\left(A_{k, j}\right) \subset B_{2(k+1) R}$, we obtain

$$
\begin{aligned}
\int_{\mathbb{R}^{n}} e^{-w_{j}} & =\sum_{k} \int_{\operatorname{Tr}\left(A_{k, j}\right)} e^{-w_{j}} \\
& \leq \sum_{k} e^{-m_{j}-k} \operatorname{Vol}\left(\operatorname{Tr}\left(A_{k, j}\right)\right) \\
& \leq \operatorname{Vol}\left(S^{n-1}\right) \sum_{k} e^{-m_{j}-k} 2(k+1) R^{n} \\
& \leq C_{n} e^{-m_{j} / 2}
\end{aligned}
$$

But, on another hand, we also have thanks to (34) and by transformation $y=$ $D w_{j+1}(x)$,

$$
\int_{\mathbb{R}^{n}} e^{-w_{j}} d x=e^{c_{X}} \int_{\Omega^{*}} e^{\sum_{l} c_{l} y_{l}} d y=C_{0} .
$$

This gives the expected inequality.

Lemma 4.4. Let $x_{j, 0}$ be the minimum point of $w_{j}$. Then there exists $C>0$ such that $\left|x_{j, 0}\right|<C$ for all $j$.

Proof. The fact that $\left|D w_{j}\right| \leq \sup _{x \in \Omega^{*}}|x|$, and the previous lemma gives the existence of $R^{\prime}$ independent of $j$ such that $\inf _{\partial B_{R^{\prime}}\left(x_{j, 0}\right)} w_{j} \leq m_{j}+1$. By convexity,

$$
\left|D w_{j}(x)\right| \geq 1 / R^{\prime}
$$

in $\mathbb{R}^{n} \backslash B_{R^{\prime}}\left(x_{j, 0}\right)$. Hence with (35), for any $\epsilon>0$, there exists $R_{\epsilon}$ sufficiently large such that

$$
\int_{\mathbb{R}^{n} \backslash B_{R_{\epsilon}}\left(x_{j, 0}\right)} e^{-w_{j}} d x \leq C_{1} \int_{\mathbb{R}^{n} \backslash B_{R_{\epsilon}}\left(x_{j, 0}\right)} e^{\left|x-x_{j, 0}\right| / R^{\prime}}<\epsilon
$$


where $R_{\epsilon}$ is independent of $j$.

Now, assume that $\left|x_{j, 0}\right|$ is not bounded. For any $\epsilon>0$, there exists a large constant $C>0$ such that if $\left|x_{j, 0}\right|>C$ then,

$$
\frac{\partial w_{j+1}}{\partial \xi}>\frac{1}{2} \inf _{x \in \partial \Omega^{*}}|x|
$$

for $x \in B_{R_{\epsilon}}\left(x_{j, 0}\right)$ and where we have set $\xi=\frac{x_{j, 0}}{\left|x_{j, 0}\right|}$. This can be viewed by considering the restriction of $w_{j+1}$ on the ray $\overrightarrow{O x_{j, 0}}$ and by its convexity together with the fact that $D w_{j+1}\left(\mathbb{R}^{n}\right)=\Omega^{*} \ni\{0\}$. Now with (35),

$$
\int_{B_{R_{\epsilon}}\left(x_{j, 0}\right)} \frac{\partial w_{j+1}}{\partial \xi} e^{-w_{j}} \geq \frac{C_{0}}{4} \inf _{x \in \partial \Omega^{*}}|x| .
$$

Furthermore, we have

$$
\left|\int_{\mathbb{R}^{n} \backslash B_{R_{\epsilon}}\left(x_{j, 0}\right)} \frac{\partial w_{j+1}}{\partial \xi} e^{-w_{j}}\right| \leq\left(\sup _{x \in \Omega^{*}}|x|\right)\left|\int_{\mathbb{R}^{n} \backslash B_{R_{\epsilon}}\left(x_{j, 0}\right)} e^{-w_{j}}\right| \leq \epsilon \sup _{x \in \Omega^{*}}|x|
$$

With (37) and (38), one obtains for $\epsilon$ sufficiently small that

$$
\int_{\mathbb{R}^{n}} \frac{\partial w_{j+1}}{\partial \xi} e^{-w_{j}} d x>0
$$

Now on another hand by (34) and (32),

$$
\begin{aligned}
0 & =\int_{\Omega^{*}} y_{i} e^{\sum_{l=1}^{n} c_{l} y_{l}} d y \\
& =\int_{\mathbb{R}^{n}} \frac{\partial w_{j+1}}{\partial x_{i}} e^{\sum_{l} c_{l} \frac{\partial w_{j+1}}{\partial x_{l}}} \operatorname{det}\left(w_{j+1}\right) d x \\
& =e^{-c_{X}} \int_{\mathbb{R}^{n}} \frac{\partial w_{j+1}}{\partial x_{i}} e^{-w_{j}} d x
\end{aligned}
$$

which leads us to $\int_{\mathbb{R}^{n}} \frac{\partial w_{j+1}}{\partial \xi} e^{-w_{j}}=0$ and hence a contradiction with (39). Thus, $\left|x_{j, 0}\right|$ has to be bounded.

Lemma 4.5. One has the upper bound

$$
\sup _{M} \Phi_{j}<C^{\prime}
$$

for a constant $C^{\prime}$ independent of $j$.

Proof. As explained in the proof of [WZ, Lemma 3.4], by convexity, it is sufficient to give an upper bound of $\Phi_{j}(0)$. Since $\left|x_{j, 0}\right|<C$ by Lemma 4.4 and $\left|D w_{j}\right| \leq \sup _{x \in \Omega^{*}}|x|$, we obtain $w_{j}(0)<C^{\prime}$. With (35), we get $\left|w_{j}(0)\right|$ bounded and hence $\left|\Phi_{j}(0)\right|$ bounded.

Lemma 4.6. One has the lower bound

$$
\inf _{M} \Phi_{j}>C^{\prime \prime}
$$

for a constant $C^{\prime \prime}$ independent of $j$. 
Proof. Let $\left\{p^{(i)}\right\}_{i=1, . ., m}$ denote the vertices of $\Omega^{*}$ and define

$$
\bar{v}(x)=\max _{i=1, . ., m}\left\{\left\langle x, p^{(i)}\right\rangle\right\} .
$$

Then the graph of $\bar{v}$ is a convex cone with vertex at the origin and is an asymptotical cone of the graph of $w_{0}$, i.e one can check that the following inequality is satisfied

$$
\left|\bar{v}-w_{0}\right| \leq c_{0}
$$

Let's denote

$$
z_{j}(r)=\sup _{|x|=r}\left(\bar{v}-w_{j}\right)(x)
$$

and assume that the supremum is attained at $p_{r, j}$. Then with $\xi=\frac{p_{r, j}}{\left|p_{r, j}\right|}$, we get

$$
z_{j}^{\prime}(r) \leq \partial_{\xi}\left(\bar{v}-w_{j}\right)\left(p_{r, j}\right) .
$$

Claim. There exists $r_{0}$ independent of $j$ such that for $r>r_{0}, z_{j}^{\prime}(r) \leq A r^{-2}$ for a constant $A$ independent of $j$.

If the claim is proved then by integration, $\sup _{x \in \mathbb{R}^{n}}\left(\bar{v}-w_{j}\right)$ is bounded independently of $j$ and the lemma is proved. Let's now prove the claim. Let $F^{(k)}$ be the faces of the graph of $\bar{v}$ and suppose that

$$
F^{(1)} \subset\left\{x_{1}>c_{0}^{\prime}|\hat{x}|\right\} \cap\left\{x_{n+1}=0\right\}
$$

which is possible by a change of variables ( $c_{0}^{\prime}$ is here a well-chosen positive constant and $\left.\hat{x}=\left(x_{2}, \ldots, x_{n}\right)\right)$. As explained in [WZ, Lemma 3.5], if the claim does not hold, there would exist a point $q_{r, j}$ in $\left\{x_{1}=\frac{1}{2} p_{r, j}\right\}$ such that

$$
w_{j}\left(q_{r, j}\right)=\inf \left\{w_{j}(x): x \in F^{(1)} \cap\left\{x_{1}=\frac{1}{2} p_{r, j}\right\}\right\} .
$$

It follows from (40) that for a certain constant $c_{0}^{\prime \prime}$,

$$
w_{j}\left(q_{r, j}\right)-w_{j}\left(p_{r, j}\right) \geq \frac{c_{0}^{\prime \prime}}{r} .
$$

Define for a convex function $f$ the set

$$
N_{f}(x)=\left\{p \in \mathbb{R}^{n}: f(y) \geq f(x)+p \cdot(y-x) \quad \forall y \in \mathbb{R}^{n}\right\}
$$

and for a set $S, N_{f}(S)=\cup_{x \in S} N_{f}(x)$. Then, for $\psi$ defined on $\mathbb{R}^{n}$ such that its graph is a convex cone with $\psi\left(p_{r, j}\right)=w_{j}\left(p_{r, j}\right)$ and $\psi=w_{j}$ on $\left\{x \in \mathbb{R}^{n}\right.$ : $\left.w_{j}(x)=w_{j}\left(q_{r, j}\right)\right\}$, one has

$$
N_{\psi}\left(S_{0}\right) \subset N_{w_{j}}\left(S_{0}\right)
$$

where we have defined $S_{0}=\left\{x \in \mathbb{R}^{n}: w_{j}(x)<w_{j}\left(q_{r, j}\right)\right\}$. From (42), one gets

$$
\operatorname{dist}\left(0, S_{0}\right) \geq c_{2} r
$$

and by (36) applied at step $j-1$, we obtain for $x \in S_{0}$ the existence of a constant $c_{3}>0$ such that

$$
w_{j-1}(x) \geq c_{3}|x|-C \geq c_{2} r-C .
$$


But now, with (34), there exists a positive constant $c_{3}^{\prime}$ such that

$$
\operatorname{Vol}\left(N_{w_{j}}\left(S_{0}\right)\right) \leq \int_{S_{0}} \operatorname{det}\left(w_{j}\right) \leq c_{3} \operatorname{Vol}\left(S_{0}\right) e^{-c_{3}^{\prime} r}
$$

and on another hand, with (41) and (42), there exists $c_{4}$ such that

$$
\operatorname{Vol}\left(N_{\psi}\left(S_{0}\right)\right) \geq \frac{c_{4}}{r^{n+1}}
$$

which leads us to the expected contradiction because of the inclusion (43).

Once the $\mathrm{C}^{0}$ estimate in hand for $\Phi_{j}$, one can derive $\mathrm{C}^{\infty}$ estimates by using the results of [Ya, TZ]. Finally the results of this section gives the

Theorem 3. Let $M$ be a Fano toric manifold with Kähler-Ricci soliton $\left(X, \omega_{\infty}\right)$. The iterations defined by

$$
\operatorname{Ric}\left(\omega_{j}\right)-L_{X}\left(\omega_{j}\right)=\omega_{j-1}
$$

for $j \geq 1$ and with $\omega_{0}$ given by (33), converge to a Kähler-Ricci soliton $\left(X, \omega_{\infty}\right)$. If the Futaki invariant vanishes, then the iterations converge to the KählerEinstein metric on $M$.

Remark 4.1. In fact our theorem gives another proof of the existence of KählerRicci solitons on Fano toric manifolds without using any flow or continuity method.

\section{Applications and numerical results}

\subsection{The finite dimensional picture}

In [Do3], Donaldson has introduced the notion of $\nu$-balanced metric for a fixed volume form $\nu$ and proved its existence [Do3, Prop. 4] under some very general conditions [Do3, p.10]. These metrics have the properties to solve the Calabi problem, i.e to converge towards the Kähler metric that has volume form $\nu$ in a given Kähler class.

Let us fix a volume form $\nu$ on a smooth projective manifold $M$ with a polarisation $L$, and choose $r \in \mathbb{N}$ sufficiently large such that $M$ is embedded by the holomorphic sections of $L^{r}$ in the projective space $\mathbb{P} H^{0}\left(M, L^{r}\right)$. We set $N_{r}=$ $h^{0}\left(M, L^{r}\right)$ which is finite since $M$ is compact. One defines a $\nu$-balanced metric at rank $r$ as the fixed point of the map $T_{\nu}: \operatorname{Met}\left(H^{0}\left(M, L^{r}\right)\right) \rightarrow \operatorname{Met}\left(H^{0}\left(M, L^{r}\right)\right)$,

$$
T(G)_{i, j}=\frac{N_{r}}{V o l_{L}(M)} \int_{M} \frac{\left\langle S_{i}, S_{j}\right\rangle}{\sum_{i}\left|S_{i}\right|^{2}} d \nu
$$

where $G$ is a hermitian metric of $H^{0}\left(M, L^{r}\right)$ and $\left(S_{i}\right)_{i=1, \ldots, N_{r}}$ is an orthonormal basis of $H^{0}\left(M, L^{r}\right)$ with respect to $G$. Donaldson proved that the compositions of the map $T_{\nu}$ give a convergent sequence of metrics in $\operatorname{Met}\left(H^{0}\left(M, L^{r}\right)\right.$ ) (and thus on $\operatorname{Met}\left(L^{r}\right)$ by the Fubini map FS, see [Do3, p.4, Section 2.2]). The limit is called the $\nu$-balanced metric.

Notation 5.1. For a smooth hermitian metric $h \in M e t(L)$ on the line bundle $L$, we denote $c_{1}(h) \in 2 \pi[L]$ its curvature. 
Theorem 4. Under these settings, let's call $H_{r} \in \operatorname{Met}\left(H^{0}\left(M, L^{r}\right)\right)$ the sequence of $\nu$-balanced metrics of order $r$. Then $c_{1}\left(F S\left(H_{r}\right)^{1 / r}\right)$ converge to a Kähler form $\omega_{\infty}$ in $\left[c_{1}(L)\right]$ that satisfies

$$
\omega_{\infty}^{n}=\nu
$$

Proof. To prove this theorem, we use the powerful Calabi-Yau theorem. Hence we know the existence of a Kähler form $\omega$ in $\left[c_{1}(L)\right]$ such that $\omega^{n}=\nu$. We use $\mathrm{X}$. Wang's theorem [Wa] with the trivial bundle and $L$. There is a HermitianEinstein metric on these bundles and the metrics $H_{r}$ are 'balanced' with respect to $\omega$ in the sense studied by Wang. This is due to the obvious fact that the considered bundles are Gieseker stable. Thus, one obtains directly the convergence of the sequence of metrics $F S\left(H_{r}\right)^{1 / r} \in \operatorname{Met}(L)$ to the metric $h_{L}$ with $c_{1}\left(h_{L}\right)=\omega$.

We now assume in this section that $M$ is Fano and consider the polarisation $L=-K_{M}$. We fix an integer $r$ sufficiently large such that $M$ is embedded by the holomorphic sections of $L^{r}$. Let's consider a smooth hermitian metric $h_{0}$ on $L$ with $c_{1}\left(h_{0}\right)=\omega_{0}$ and let's call $f_{\omega_{0}}$ the Ricci deviation of $\omega_{0}$. Now for each $k$, one can define a $\operatorname{Ric}^{(-k)}\left(\omega_{0}\right)^{n}$-balanced metric at rank $r$ in the following way.

Consider $\operatorname{Hilb}_{\omega_{0}}\left(h_{0}^{r}\right)$ the $L^{2}$-metric induced on the space of holomorphic sections $H^{0}\left(M, L^{r}\right)$ with respect to $\omega_{0}$, given by

$$
\operatorname{Hilb}_{\omega_{0}}(\langle,\rangle)\left(S_{i}, S_{j}\right)=\int_{M}\left\langle S_{i}, S_{j}\right\rangle e^{f_{\omega_{0}} \omega_{0}^{n}}
$$

Now, for a given hermitian metric $H_{0}$ on $H^{0}\left(M, L^{r}\right)$, we define the metric $F S\left(H_{0}\right)$ on $L^{r}$ by

$$
\sum_{i=1}^{N_{r}}\left|S_{i}\right|_{F S\left(H_{0}\right)}^{2}=\frac{N_{r}}{V}
$$

where the $\left(S_{i}\right)_{i=1, . ., N_{r}}$ form an $H_{0}$-orthonormal basis of $H^{0}\left(M, L^{r}\right)$. Then, from [Do3, Prop. 4], we know that the dynamical system $F S \circ H i l b_{\omega_{0}}$ has an attractive fixed point $h_{\omega_{0}, r}$ at rank $r$ and that the convergence of this dynamical system is exponentially fast. We obtain this way a new form

$$
\omega_{1, r}=c_{1}\left(h_{\omega_{0}, r}\right)
$$

For the second step, i.e in order to find the balanced metric $h_{\mathrm{Ric}^{-1}\left(\omega_{0}\right), r}$, we introduce the operator $H i l b_{\omega_{1, r}}$, by

$$
\operatorname{Hilb}_{\omega_{1, r}}(\langle,\rangle)\left(S_{i}, S_{j}\right)=\int_{M}\left\langle S_{i}, S_{j}\right\rangle e^{-\varphi_{0, r}} \omega_{1, r}^{n}
$$

where $\varphi_{0, r}$ is the potential of the metric $h_{\omega_{0}, r} \in \operatorname{Met}\left(L^{r}\right)$. Iterating this procedure leads us to define at each step $k$ a dynamical system $H i l b_{\omega_{k, r}} \circ F S$ which has an attractive fixed point $h_{\omega_{k, r}} \in \operatorname{Met}\left(L^{r}\right)\left(=e^{-\varphi_{k, r}|\cdot|_{0}}\right.$ locally). Note that for a generic $k$, we have

$$
\operatorname{Hilb}_{\omega_{k, r}}(\langle,\rangle)\left(S_{i}, S_{j}\right)=\int_{M}\left\langle S_{i}, S_{j}\right\rangle e^{-\varphi_{k-1, r}} \omega_{k, r}^{n}
$$

Corollary 5.1. Under above assumptions, and for $r$ sufficiently large, the sequence $c_{1}\left(h_{\omega_{l, r}}^{1 / r}\right)$ converges when $r$ tends to infinity to the solution of the MongeAmpère equation (19) with exponential speed of convergence. 
Conjecture 1. Under above assumptions, the sequence $c_{1}\left(h_{\omega_{l, r}}^{1 / r}\right)$ converges when $l$ tends to infinity to a Kähler metric $\omega_{r}$ in the class $c_{1}(M)$ with exponential speed of convergence. If $M$ is Kähler-Einstein, then $\omega_{r}$ converges to a KählerEinstein metric.

Let's describe now how our discussion can be useful for numerical approximations of Kähler-Einstein metrics on Fano manifolds. One has to notice at this stage that we can write (44) as

$$
\operatorname{Hilb}_{\omega_{k, r}}(\langle,\rangle)\left(S_{i}, S_{j}\right)=\int_{M}\left\langle S_{i}, S_{j}\right\rangle\left(\frac{V}{N_{r}} \sum_{i}\left|\widetilde{S_{i, k-1}}\right|^{2}\right)^{-1 / r}
$$

where the $\left(\widetilde{S_{i, k-1}}\right) \in H^{0}\left(M, L^{r}\right)$ form an orthonormal basis of holomorphic sections with respect to the $L^{2}$ metric $H_{k-1, r}=H i l b_{\omega_{k-1, r}}\left(h_{\omega_{k-1, r}}\right)$ computed at the previous step, i.e the $\operatorname{Ric}^{-(k-1)}\left(\omega_{0}\right)^{n}$-balanced metric. Note that the expression (45) makes sense since the term $\left(\sum_{i}\left|\widetilde{S_{i, k-1}}\right|^{2}\right)^{-1}$ can be considered as a section of $K_{M}^{r} \otimes{\overline{K_{M}}}^{r}$.

In order to obtain the $\mathrm{Ric}^{-k}\left(\omega_{0}\right)^{n}$-balanced metric $H_{k, r}$ one needs to iterate the operator $H i l b_{\omega_{k, r}} \circ F S$, which gives a sequence $\left(H_{k, r, p}\right)_{p \in \mathbb{N}} \in \operatorname{Met}\left(H^{0}\left(M, L^{r}\right)\right)$ initialized with $H_{k, r, 0}=H_{k-1, r}$. Here, we denote $H_{k, r}=H_{k, r, \infty}$. Remark now that if one expects the algorithm to be convergent, and thus $H_{k-1, r}$ to be close to $H_{k, r}$ for large $k$ and $r$, then it is natural to assume that $H_{k-1, r, 1}$ is close to $H_{k-1, r, \infty}=H_{k, r, 0}$, i.e just one step is sufficient to get the $\mathrm{Ric}^{-k}\left(\omega_{0}\right)^{n}$-balanced metric. This justifies at least formally the definition of canonically balanced metrics for Fano manifolds that appeared in [Do3, Section 2.2.2].

Definition 5.1. Let $M$ be a Fano manifold and $r \in \mathbb{N}^{*}$ sufficiently large. For a metric $G \in \operatorname{Met}\left(H^{0}\left(M,-K_{M}^{r}\right)\right.$, we define the operator $\widetilde{T}$ on $\operatorname{Met}\left(H^{0}\left(M,-K_{M}^{r}\right)\right.$ by

$$
\widetilde{T}(G)_{i j}=\int_{M} \frac{\left\langle S_{i}, S_{j}\right\rangle}{\left(\sum_{i}\left|S_{i}\right|^{2}\right)^{1+1 / r}}
$$

for $\left(S_{i}\right) \in H^{0}\left(M,-K_{M}^{r}\right)$ a $G$-orthonormal basis. A canonically balanced metric $G_{\text {can }} \in \operatorname{Met}\left(H^{0}\left(M,-K_{M}^{r}\right)\right)$ is a fixed point of the operator $\widetilde{T}$.

The operator $\widetilde{T}$ seen as acting on Bergman type metrics is actually a finite dimensional approximation of the $\mathrm{Ric}^{-1}$ operator. Hence, when they exist a priori, we expect the behaviour of the sequence of canonically balanced metrics to be understood via the iterations of the $\mathrm{Ric}^{-1}$ operator, and thus to be related to the behaviour of the Kähler-Ricci flow. In that direction, we can show

Theorem 5. Assume that $M$ is a Fano Einstein manifold with no nontrivial holomorphic vector field. Then for $r$ sufficiently large, there exists a sequence of canonically balanced metrics $G_{c a n, r} \in \operatorname{Met}\left(H^{0}\left(M,-K_{M}^{r}\right)\right)$ such that $c_{1}\left(F S\left(G_{c a n, r}\right)\right)$ converges when $r$ tends to infinity to the Kähler-Einstein metric $\omega_{K E}$.

Proof. The proof is similar to the proof of the main result of [Do1]. Actually, a canonically balanced metric $h_{c a n, r} \in \operatorname{Met}\left(-K_{M}^{r}\right)$ satisfies that the Bergman 
kernel is constant on the manifold, i.e for all $p \in M$,

$$
\sum_{i=1}^{N_{r}}\left|S_{i}\right|_{h_{c a n, r}}^{2}(p)=\frac{N_{r}}{V}
$$

where the sections $S_{i} \in H^{0}\left(M,-K_{M}^{r}\right)$ are orthonormal with respect to the inner product

$$
<a, b>=\int_{M}\left(h_{c a n, r}\right)^{\frac{r+1}{r}} \otimes a \otimes \bar{b},
$$

and $N_{r}=\operatorname{dim} H^{0}\left(M,-K_{M}^{r}\right)$. Note that one can generalize the results of $\mathrm{Z}$. $\mathrm{Lu}[\mathrm{Lu}]$ on the asymptotic of the Bergman kernel in that case. The details will appear in a forthcoming paper where the relationship with G.I.T stability will be studied.

In our implementations we use this notion, i.e we consider the iterations of the operator $\widetilde{T}$. At each step we also choose a normalisation for the metric by fixing essentially its volume. Our tests on toric manifolds have shown that the sequence of metrics defined by (45) and (46) have similar behaviours (i.e iterating many times $H_{i l b} b_{k, r} \circ F S$ or just once) and converge to the Kähler-Einstein metric when it exists a priori. These procedures have the advantage to skip the computation of the determinant of the Fubini-Study metric as required by the original notion of balanced metric [Do1, Luo, Zha]. Therefore they are clearly much more efficient and we expect that similar methods could be developed for Einstein non Fano manifolds. Finally, remark that even in the case of $\mathbb{C P}^{1}$ with the anticanonical polarisation, the sequence of balanced and canonically balanced metrics converge at different speeds towards the Fubini-Study metric [Do3].

\subsection{The case of the projective plane blown up in three points}

Let us consider the toric Fano manifold $\mathbb{P}^{2}$ blown up in 3 (non aligned) points. From a result of J. Song, its $\alpha$-invariant is 1 and thus it possesses a KählerEinstein metric (this is also a consequence of Tian's work of classification of Einstein Del Pezzo surfaces). Let us mention that the Kähler-Einstein metric on this manifold has been very recently studied in [DHHKW] by simulating the Ricci flow with PDE techniques.

We implement our algorithms (i.e in order to find balanced and canonically balanced metrics) using the special symmetries of this manifold. The computations of the points on the manifolds are relatively quick since we are essentially reduced to a 2-dimensional real manifold and there are some symmetries given by the action of $\mathbb{Z}_{2}$ (reflections) and $\mathbb{Z}_{6}$ (rotations). The fan of this toric variety is given by the six rays spanned by

$v_{0}=(1,0), v_{1}=(1,1), v_{2}=(0,1), v_{3}=(-1,0), v_{4}=(-1,-1), v_{5}=(0,-1)$.

and as it is well known the polytope is actually the hexagon. There are different ways to choose the points on this toric manifold but we decided to just generate the points on one of the 6 affine charts associated to the cone formed by pairs of rays $\left(v_{i}, v_{i+1}\right)$ (i.e. by defining a certain cut-off function). 
Our program is written in $\mathrm{C}++$ (compiler gcc 3.4.6) and can be launched essentially with 4 different algorithms. For each algorithm we print the scalar curvature of the new computed metric computed at each point of the manifold. This gives a picture with different colours and one can easily see after some iterations that the scalar curvature varies essentially for the points close to the edges. The computation of the scalar curvature is possible with exact precision (up to the machine precision) since our metrics are algebraic. It has the disavantage to take time since it involves derivatives of order 4 , but on the other hand it gives a visual output of our work, see Figure (1). Some animated pictures generated by the program and the program itself can be downloaded from the website of the author ${ }^{2}$. Despite of this loss of time, all the four algorithms (for the given parameters below) can be run in 1 minute or less on a decent desktop computer. This proves the efficiency of the methods and let us hope that it is possible to compute Kähler-Einstein metric on 3 -folds with few symmetries. We now describe the results for each algorithm.

For the computation of the balanced metric as defined in [Do1, Do2, Do3], we choose the parameter $r=8$ (see Section 5.1) and compute approximatively $10^{4}$ points. After 50 iterations, the average scalar curvature on the manifold is 0.95 and the maximum error is $16 \%$.

For the computation of the canonically balanced metric as defined in [Do3] or our discretization of the Ricci flow (45), we fix again $r=8$ and compute approximatively $5 \cdot 10^{4}$ points. After 35 iterations, the average scalar curvature on the manifold is now 0.99 and the maximum error is less than $4 \%$.

Finally we try to improve the our first two algorithms by using the metric at rank $r$ to compute the metric at rank $r+1$. This is based on a very simple argument that we describe now. If one knows the balanced $h_{r} \in \operatorname{Met}\left(L^{r}\right)$ (or canonical balanced metric) at rank $r$, then using the asymptotic of the Bergman function for higher tensor powers (see [Lu] for details) one can write on this surface

$$
\frac{N_{r}}{V}=\sum_{i}\left|S_{i}\right|_{h_{r}}^{2}(p)=r^{2}+r \frac{\operatorname{scal}\left(c_{1}\left(h_{r}\right)\right)(p)}{2}+\Gamma(p)+O(1 / r)
$$

where $\Gamma(p)$ is a certain function which in fact is an algebraic expression of the curvature of $c_{1}\left(h_{r}\right)$ and its derivatives. Here $\left(S_{i}\right)_{i=1, . ., N_{r}}$ is an orthonormal basis of $H^{0}\left(M, L^{r}\right)$ with respect to the $L^{2}$-metric corresponding to the choice of our algorithm. Once we have computed $h_{r}$, it is clear that we can deduce the value of $\Gamma$ at each point of the manifold. Now at rank $r+1$, we look for a metric $\tilde{h}_{r+1}$ such that

$$
\begin{aligned}
\sum_{i=1}^{N_{r+1}}\left|S_{i}\right|_{\tilde{h}_{r+1}}^{2}(p) & =\frac{N_{r+1}}{V}+\Gamma(p) \\
& =\frac{1}{V}\left(N_{r+1}+\frac{r}{2}\left(1-\operatorname{scal}\left(c_{1}\left(h_{r}\right)\right)(p)\right)\right)
\end{aligned}
$$

with respect to the corresponding $L^{2}$-metric. Roughly speaking, it corresponds to force the algorithm to get a metric with constant scalar curvature up to an

\footnotetext{
${ }^{2}$ http://www.ma.ic.ac.uk/ jkeller/Julien-KELLER-progs.html Some other programs for other Fano Einstein surfaces will be available at this address.
} 

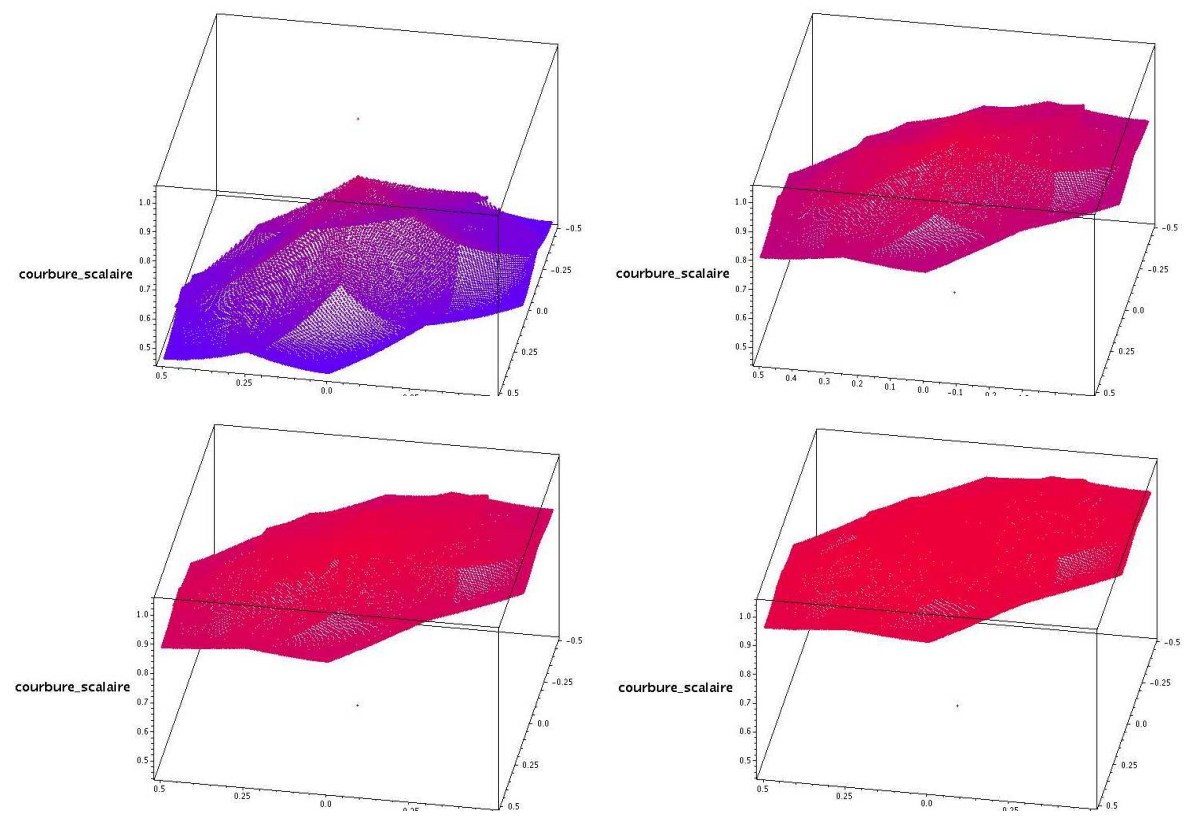

Figure 1: Iterations no. 10,16,20,40 for the canonically balanced metric

error of size $O\left(1 /(r+1)^{2}\right)$ (instead of only $O(1 /(r+1))$ for the balanced metric). We apply also the same trick for the canonically balanced metrics. Finally we call these new sequence of metrics as 1-step recursively balanced (1-s.bal in short) or 1-step recursively canonically balanced (1-s.c.bal in short).

The advantage of this method is that it is particularly simple (at least for dimension 2) to program it, since we have already coded the computation of the scalar curvature. On the other hand it asks of course a little bit more time of computation at each step. We give now an overview of the results for $r=4$, a choice of $10^{4}$ points on the manifolds and 15 iterations for each algorithm.

\begin{tabular}{l||c|c|c|c} 
Method $(r=4)$ & Balanced & 1-s.bal & Can. balanced & 1-s.c.bal \\
Avg scalar curvature & 0.786 & 0.888 & 0.949 & 0.984 \\
Max scalar curvature & 1.079 & 1.041 & 1.012 & 1.041 \\
Min scalar curvature & 0.618 & 0.717 & 0.820 & 0.867 \\
Time (sec) & 9.1 & 16.1 & 8.0 & 15.5
\end{tabular}

As an example, we obtain Figure 1 p.23 for the canonically balanced metric with $r=12$ and various iterations. One can see that for 40 iterations we get a metric with scalar curvature almost equal to 1 everywhere. Using this metric, one can find numerical approximations ${ }^{3}$ of geodesics for the Kähler-Einstein metric. It seems to give a numerical evidence that the geodesic equations on this manifold form an integrable system.

\footnotetext{
${ }^{3}$ http://www.ma.ic.ac.uk/ jkeller/Julien-KELLER-progs.html
} 


\section{Iteration for other classes}

\subsection{The case of negative first Chern class}

Assume now that $K_{M}>0$ i.e that $M$ has negative first Chern class. It is well known by [Ya] that such manifolds have a unique Kähler-Einstein metric $\omega_{K E}$ such that

$$
\operatorname{Ric}\left(\omega_{K E}\right)=-\omega_{K E} .
$$

Let Ric $\omega+\omega=\sqrt{-1} \partial \bar{\partial} h$ with $\frac{1}{V} \int_{M} e^{h} \omega^{n}=1$. In that case, the naive discretization of the normalised Kähler-Ricci flow leads us to define the iteration scheme by

$$
-\operatorname{Ric} \omega_{l}=2 \omega_{l}-\omega_{l-1} .
$$

This can be rewritten in terms of the following Monge-Ampère equations

$$
\omega_{\Sigma_{j=1}^{l} \varphi_{j}}^{n}=e^{2 \varphi_{l}-\varphi_{l-1}} \omega_{\sum_{j=1}^{l-1} \varphi_{j}}^{n}, \quad \frac{1}{V} \int_{M} e^{2 \varphi_{l}-\varphi_{l-1}} \omega_{l-1}^{n}=1, \quad \forall l \in \mathbb{N} .
$$

and we will denote as previously $\Phi_{l}=\sum_{j=1}^{l} \varphi_{l}$ and $\omega_{l}=\omega+\sqrt{-1} \partial \bar{\partial} \Phi_{l}$. Note that by the general results on Monge-Ampère equations [Ko], for any $l$, there exists a smooth solution $\varphi_{l}$ of (48) and thus the iteration scheme is well-defined. By concavity of the log applied to (48), we have directly

Lemma 6.1. Let $\mu=-1$. Then

$$
E_{0}\left(\omega_{l-1}, \omega_{l}\right)=-J\left(\omega_{l}, \omega_{l+1}\right)+\frac{1}{V} \int_{M}\left(2 \varphi_{l}-\varphi_{l-1}\right) \omega_{l-1}^{n} \leq 0
$$

i.e the iterations (48) decrease the functional $E_{0}$.

Proposition 6.1. Let $p \in(1, \infty)$. There exists a constant $C_{1}$ depending only on $p, M$ and $\omega$ such that

$$
\left\|\Phi_{l}\right\|_{L^{\infty}(M, \omega)} \leq C_{1}, \quad \forall l \in \mathbb{N} .
$$

Proof. Let $p \in(1, \infty)$. According to the work of [Ko] (or [Bl] if $p \in(2, \infty)$ which will suffice for the proof) and in view of (48) it suffices to prove that

$$
\left\|e^{h+2 \Phi_{l}-\Phi_{l-1}}\right\|_{L^{p}(M, \omega)} \leq C_{2}, \quad \forall l \in \mathbb{N},
$$

for $C_{2}=C(M, \omega, p)$. From the normalisation in (48) it follows that $\frac{1}{V} \int_{M}(h+$ $\left.2 \Phi_{l}-\Phi_{l-1}\right) \omega^{n} \leq 0$ where $h$ denotes the Ricci deviation of $\omega$. In particular $\sup _{M}\left(h+2 \Phi_{l}-\Phi_{l-1}\right) \leq-\frac{1}{V} \int_{M}\left(h+2 \Phi_{l}-\Phi_{l-1}-\sup _{M}\left(h+2 \Phi_{l}-\Phi_{l-1}\right)\right) \omega^{n}$. Therefore

$$
\begin{aligned}
\frac{1}{V} \int_{M} e^{p\left(h+2 \Phi_{l}-\Phi_{l-1}\right)} \omega^{n} & \leq e^{p \sup _{M}\left(h+2 \Phi_{l}-\Phi_{l-1}\right)} \\
& \leq e^{-p \frac{1}{V} \int_{M}\left(h+2 \Phi_{l}-\Phi_{l-1}-\sup _{M}\left(h+2 \Phi_{l}-\Phi_{l-1}\right)\right) \omega^{n}} \\
& \leq e^{p \operatorname{osc}_{M} h} e^{-p \frac{1}{V} \int_{M}\left(2 \Phi_{l}-\Phi_{l-1}-\sup _{M}\left(2 \Phi_{l}-\Phi_{l-1}\right)\right) \omega^{n}}
\end{aligned}
$$

Let $G$ be the Green function for $\Delta=\Delta_{\bar{\partial}}$ satisfying $\int_{M} G(x, y) \omega^{n}(y)=0$. Since $\omega_{0}+2 \Phi_{l}-\Phi_{l-1}=2 \omega_{l}-\omega_{l-1}>0$ the Green formula gives

$$
\begin{aligned}
& \frac{1}{V} \int_{M}\left(\sup _{M}\left(2 \Phi_{l}-\Phi_{l-1}\right)-\left(2 \Phi_{l}-\Phi_{l-1}\right)\right) \omega^{n} \\
& \quad=-\frac{1}{V} \int_{M} G\left(x_{0}, y\right) \Delta\left(2 \Phi_{l}-\Phi_{l-1}\right) \omega^{n}(y) \leq n A
\end{aligned}
$$


where $A=-\inf _{M \times M} G(x, y)$ and $x_{0} \in M$ satisfies $\left(2 \Phi_{l}-\Phi_{l-1}\right)\left(x_{0}\right)=\sup _{M}\left(2 \Phi_{l}-\right.$ $\left.\Phi_{l-1}\right)$. Therefore $C_{2}=e^{p\left(\operatorname{osc}_{M} h+n A\right)}$.

Combining the previous proposition and Lemma 6.1, we obtain

Corollary 6.1. Let $M$ be a manifold with $K_{M}>0$. Then, the sequence of Kähler metrics defined by (47) converges to the Kähler-Einstein metric $\omega_{K E}$ on $M$.

Actually, another iteration scheme is also very natural, especially if we assume the metrics $\omega_{l}$ and $\omega_{l+1}$ from (47) to be very 'close' for a large $l$. We can define the iteration scheme

$$
-\operatorname{Ric} \widetilde{\omega}_{l}=\widetilde{\omega}_{l-1}
$$

which means that we solve at each step the following Monge-Ampère equations

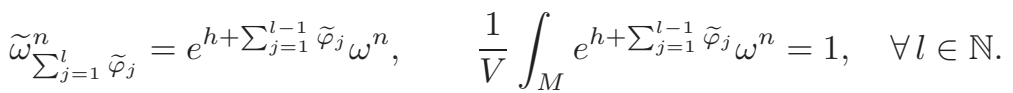

Let's call $\widetilde{\Phi}_{l}=\sum_{j=1}^{l} \widetilde{\varphi}_{j}$. Up to our knowledge, it is not clear whether the iteration scheme (50) decreases any functional. Now, one can check that the proof of Proposition 6.1 can be adapted to that case without must change, i.e one has straightforward a $C^{0}$-estimate of the potentials $\widetilde{\Phi}_{l}$ and thus convergence up to extraction of a subsequence. On another hand, the iteration scheme (50) is more natural from the finite dimensional perspective as we shall explain now.

Similarly to the notion of canonically balanced metric introduced by (46) in Section 5.1, one can define a notion of canonically balanced for manifolds with negative first Chern class. Indeed, it turns out to consider the iteration procedure defined by the iterations of the map $\widetilde{T}=\widetilde{H i l b} \circ F S$ where

$$
\widetilde{\operatorname{Hilb}}(h)\left(S_{i}, S_{j}\right)=\int_{M} h^{\frac{k-1}{k}} \otimes S_{i} \otimes \overline{S_{j}}
$$

for $h \in \operatorname{Met}\left(K_{M}^{k}\right)$ and $S_{i} \in H^{0}\left(M, K_{M}^{k}\right)$. Here we see $h^{\frac{k-1}{k}}$ as an element of $K_{M}^{1-k} \otimes{\overline{K_{M}}}^{1-k}$. From our previous discussion p.20 Section 5.1, one can expect naturally the iterates of $\widehat{T}$ to approximate in the space $H^{0}\left(M, K_{M}^{k}\right)$ the discretization of the normalised Kähler-Ricci flow given by (50).

As explained in [Ts], another remarkable fact is that one can define an iterative procedure in higher dimensional spaces, i.e by defining now the maps $\widehat{T}=$ $\widehat{H i l b} \circ F S: \operatorname{Met}\left(K_{M}^{k}\right) \rightarrow \operatorname{Met}\left(K_{M}^{k+1}\right)$ where

$$
\widehat{\operatorname{Hilb}}(h)\left(S_{i}, S_{j}\right)=\int_{M} h \otimes S_{i} \otimes \overline{S_{j}} .
$$

Let's define $h_{k}=\widehat{T}^{(k)}\left(h_{0}\right)$ for a metric $h_{0} \in \operatorname{Met}\left(K_{M}^{k_{0}}\right)$ for $k_{0}$ sufficiently large. As observed by Weinkove and Song [SW2, Theorem 1], one has a uniform convergence for $k \rightarrow+\infty$ of the metrics $h_{k}^{\frac{1}{k+k_{0}}}$ to the smooth hermitian metric $h_{K E} \in \operatorname{Met}\left(K_{M}\right)$ such that $c_{1}\left(h_{K E}\right)=\omega_{K E}$ is the Kähler-Einstein metric on $M$. Nevertheless this method does not seem to have numerical applications straightforward since it involves to consider higher rank matrices (and thus their inverses). 
Remark 6.1. One can also derive an iterative scheme for manifolds with trivial canonical bundle. This leads to a refinement of the notion of $\nu$-balanced metric [Do3], and is useful for our numerical study of Ricci-flat metrics on Calabi-Yau threefolds. This will be addressed in a forthcoming paper.

\subsection{The case of non-canonical classes}

Let $\omega \in K a_{\Omega}$ be a Kähler representative of an arbitrary class $\Omega$ in the Kähler cone of a Fano manifold $M$. Let $\sigma \in K a_{c_{1}}^{(0)}$ be a representative of $c_{1}(M)$. By the Calabi-Yau theorem there exists a unique Kähler representative $\omega_{1}$ of $\Omega$ whose Ricci form equals $\sigma$. Define a map

$$
\operatorname{Ric}_{\Omega}^{(-1)}: K a_{c_{1}}^{(0)} \rightarrow K a_{\Omega}
$$

by $\operatorname{Ric}_{\Omega}^{(-1)} \sigma:=\omega_{1}$ (when appropriate norms are chosen, it defines again a homeomorphism of Banach spaces). We may therefore define an operator $\operatorname{Ric}_{[\omega]}$ on $K a_{\Omega}$ by

$$
\operatorname{Ric}_{[\omega]}:=\operatorname{Ric}_{\Omega}^{(-1)} \circ \operatorname{Ric} \circ \text { Ric. }
$$

This gives rise to an iteration on $\Omega$ by setting $\operatorname{Ric}_{\Omega}^{(k)}:=\operatorname{Ric}_{\Omega}^{(-1)} \circ \operatorname{Ric}^{(k)} \circ \operatorname{Ric}$ for each $k \in \mathbb{Z}$. Note that this induces again a filtration on $K a_{\Omega}$ defined by $K a_{\Omega}^{(k)}:=\left\{\omega \in K a_{\Omega}: \operatorname{Ric} \omega \in K a_{c_{1}}^{(k)}\right\}$.

From what we have proved previously, we expect that the orbits of the new dynamical systems converge, if and only $M$ is Kähler-Einstein, to a Kähler representative which is characterised by the property that its Ricci form is KählerEinstein. For $\Omega \neq c_{1}(M)$, one can ask how is this dynamical system related to the study of the space $K a_{\Omega}$. In particular, it would be interesting to relate this to the existence problem of extremal metrics for Kähler classes near $c_{1}(M)$.

Acknowledgements. The author is extremely grateful to S.K. Donaldson for enlightening conversations and his constant encouragement. He also thanks R. Bunch for his very useful help with $\mathrm{C}++$ programs. This work has also benefited from stimulating discussions with X.X. Chen, D. Panov, D.H. Phong, S. Simanca, G. Szekelyhidi and R.P. Thomas. Finally he thanks P. Eyssidieux for his support throughout the years.

\section{References}

[Au1] T. Aubin, Réduction du cas positif de l'équation de Monge-Ampère sur les variétés kählériennes compactes à la démonstration d'une inégalité, J. of Functional Analysis 57 (1984), 143-153.

[Au2] T. Aubin, Métriques d'Einstein-Kähler et exponentiel des fonctions admissibles, J. of Functional Analysis 88 (1990), 385-394. 
[Au3] T. Aubin, Some nonlinear problems in Riemannian Geometry, Springer, (1998).

[Ba] S. Bando, The K-Energy Map, Almost Kähler-Einstein Metrics and an Inequality of the Miyaoka-Yau Type, Tôku Mathematical Journal 39 (1987), 231-235.

[BM] S. Bando \& T. Mabuchi, Uniqueness of Kähler-Einstein Metrics Modulo Connected Group Actions, in Algebraic Geometry, Sendai, 1985, Advanced Studies in Pure Mathematics 10 (1987), 11-40.

[Be] B. Berndtsson, Bergman kernels related to Hermitian line bundles over compact complex manifolds, Explorations in complex and Riemannian geometry, Contemp. Math., 332, Amer. Math. Soc., Providence, RI, (2003), 1-17.

[Bl] Z. Blocki, Uniqueness and Stability for the Complex Monge-Ampère Equation on Compact Kähler Manifolds, Indiana University Mathematics Journal 52 (2003), 1697-1701.

[Ch] X.X. Chen, On the lower bound of the Mabuchi energy and its application, International Math. Research Notices 12 (2000), 607-623.

[CT] X.X. Chen \& G. Tian, Ricci flow on Kähler-Einstein surfaces, Inventiones Mathematicæ 147 (2002), 487-544.

[Di] W-Y. Ding, Remarks on the existence problem of positive KählerEinstein metrics, Math. Annalen 282 (1988), 463-471.

[Do1] S.K. Donaldson, Scalar curvature and projective embeddings I, J. Diff. Geom. 59 (2001), 479-522.

[Do2] S.K. Donaldson, Scalar curvature and projective embeddings II, Quaterly Jour. Math 56 (2005), 345-356.

[Do3] S.K. Donaldson, Some numerical results in complex differential geometry, arXiv (2005).

[DHHKW] C. Doran \& M. Headrick \& C. P. Herzog \& J. Kantor \& T. Wiseman, Numerical Kaehler-Einstein metric on the third del Pezzo, arXiv:hep-th/0703057v2 (2007).

[Ko] S. Kolodziej, The complex Monge-Ampère equation and pluripotential theory, Memoirs of the American Mathematical Society 178 (2005), no. 840.

[Lu] Z. Lu, On the lower order terms of the asymptotic expansion of Tian-Yau-Zelditch, Amer. J. Math 122 (2000), 235-273.

[Luo] H. Luo, Geometric criterion for Gieseker-Mumford stability of polarized manifolds, J. Diff. Geom. 49 (1998), 577-599.

[Ma] T. Mabuchi, K-energy maps integrating Futaki invariants, Tôhuku Mathematical Journal 38 (1986), 575-593. 
[Na] A. Nadel, On the Absence of Periodic Points for the Ricci Curvature Operator Acting on the Space of Kähler Metrics, in Modern Methods in Complex Analysis: The Princeton Conference in Honor of Gunning and Kohn, Annals of Mathematics Studies 137 (1995), $273-282$.

[Pa] N. Pali, Characterization of Einstein-Fano manifolds via the KählerRicci flow, arXiv:math/0607581v2 [math.DG] (2007).

[PSSW] D.H. Phong, J. Song, J. Sturm, B. Weinkove, The Moser-Trudinger inequality on Kähler-Einstein manifolds, arXiv:math.DG/0604076 (2006).

[Ru] Y. Rubinstein, Some discretizations of geometric evolution equations and the Ricci iteration on the space of Kahler metrics, I, arXiv:0709.0990 (2007).

[Si] Y-T. Siu, Lectures on Hermitian-Einstein metrics for stable bundles and Kähler-Einstein metrics, Birkhäuser, 1987.

[SW1] J. Song \& B. Weinkove, Energy Functionals and Canonical Kähler Metrics, preprint, arxiv:math.DG/0505476.

[SW2] J. Song \& B. Weinkove, Constructions of Kähler-Einstein metrics with negative scalar curvature, preprint, arxiv:math.DG/0704.1005.

[Ti0] G. Tian, On Kähler-Einstein metrics on certain Kähler manifolds with $C_{1}(M)>0$, Inventiones Math. 89 (1987), 225-246.

[Ti1] G. Tian, Canonical Metrics in Kähler Geometry, Lectures in Mathematics ETH Zürich, Birkhäuser, 2000.

[TZ] G. Tian \& X. Zhu, Uniqueness of Kähler-Ricci solitons, Acta Math. 184 (2000) 271-305.

[Ts] H. Tsuji, Dynamical constructions of Kähler-Einstein metrics, Arxiv:math.AG/0606626 (2006).

[Ya] S-T. Yau, On the Ricci Curvature of a Compact Kähler manifold and the Complex Monge-Ampère equation, I, Communications in Pure and Applied Mathematics 31 (1978), 339-411.

[Wa] X. Wang, Canonical metrics on stable vector bundles, Comm. Anal. Geom. 13 (2005), 253-285.

[WZ] X-J. Wang \& X. Zhu, Kähler-Ricci solitons on toric manifolds with positive first Chern class, Advances in Math. 188 (2004), 87-103.

[Zha] S. Zhang, Heights and reductions of semi-stable varieties, Compositio Math. 104 (1996), 77-105.

[Zhu1] X. Zhu, Kähler-Ricci soliton type equations on compact complex manifolds with $c_{1}(M)>0$, J. Geom. Anal. 10 (2000), 759-774.

[Zhu2] X. Zhu, Kähler-Ricci flow on a toric manifold with positive first Chern class, arXiv:math.DG/0703486 (2007). 
Julien Keller

ImPerial College, LONDON

J.KELLER@IMPERIAL.AC.UK 